\title{
Adherence to Masculine Norms and Perceived Paternal Nurturance as Predictors of SelfCompassion
}

\author{
Steven J. Craig
}

Follow this and additional works at: https://researchrepository.wvu.edu/etd

\section{Recommended Citation}

Craig, Steven J., "Adherence to Masculine Norms and Perceived Paternal Nurturance as Predictors of SelfCompassion" (2018). Graduate Theses, Dissertations, and Problem Reports. 7170.

https://researchrepository.wvu.edu/etd/7170

This Dissertation is protected by copyright and/or related rights. It has been brought to you by the The Research Repository @ WVU with permission from the rights-holder(s). You are free to use this Dissertation in any way that is permitted by the copyright and related rights legislation that applies to your use. For other uses you must obtain permission from the rights-holder(s) directly, unless additional rights are indicated by a Creative Commons license in the record and/ or on the work itself. This Dissertation has been accepted for inclusion in WVU Graduate Theses, Dissertations, and Problem Reports collection by an authorized administrator of The Research Repository @ WVU.

For more information, please contact researchrepository@mail.wvu.edu. 
Adherence to Masculine Norms and Perceived Paternal Nurturance as Predictors of SelfCompassion

Steven J. Craig, M.Ed.

Dissertation submitted to the College of Education and Human Services at West Virginia University

in partial fulfillment of the requirements

for the degree of

Doctor of Philosophy in

Counseling Psychology

Jeffrey Daniels, Ph.D., Chair G. David Allen, Ph.D.

Shane Chaplin, Ph.D.

Monica Leppma, Ph.D. Christine Schimmel, Ed.D.

Department of Counseling, Rehabilitation Counseling, and Counseling Psychology

Morgantown, West Virginia 2018

Keywords: masculinity, self-compassion, paternal nurturance, fatherhood Copyright 2018 Steven Craig 


\section{Abstract \\ Adherence to Masculine Norms and Perceived Paternal Nurturance as Predictors of Self- Compassion}

\section{Steven J. Craig}

Previous researchers have examined the relationship between adherence to masculine norms and self-compassion as well as the impact of parental warmth on self-compassion. However, no study to date has specifically examined how perceptions of paternal nurturance and adherence to masculine norms impact levels of self-compassion in college-aged males. Furthermore, findings regarding the relationship between adherence to traditional gender roles and self-compassion have not been well established. The purpose of the current quantitative study was to ascertain whether greater adherence to traditional United States' masculine norms and levels of perceived paternal nurturance contribute to levels of self-compassion in college-aged males at West Virginia University. Data were collected via self-report measures in order to better understand whether the first two variables predicted levels of self-compassion. A hierarchical multiple regression was used to ascertain whether perceptions of paternal nurturance would explain a significant amount of variance in levels of self-compassion after controlling for adherence to masculine norms. Findings demonstrated that higher levels of adherence to masculine norms predicted lower levels of self-compassion and that higher levels of perceived paternal nurturance predicted lower levels of self-compassion. Overall, the hierarchical regression analysis showed that perceived paternal nurturance did explain a significant amount of variance in levels of selfcompassion while controlling for levels of adherence to masculine norms, though not in the direction initially hypothesized. The implications based upon the results and future directions for study are discussed. 


\section{Dedication}

\section{To Mike}

... a true friend is more than just someone with whom we share common values and who accepts us for what we are. Such a friend is someone whom we can trust to refine our understanding of what it means to live...

-Stephen Batchelor 


\section{Acknowledgments}

I would first like to thank Dr. Daniels for your encouragement, support, and mentorship throughout this entire process. Your dedication to the field of counseling psychology has been an inspiration to me and I am truly grateful for having you be a part of my journey. I would also like to thank Dr. Bartee for all that you have done for me throughout my time in the program. You've helped me to not only see this project from different perspectives, but also my life and experiences in the world. To Dr. Allen, Shane, Dr. Leppma, and Dr. Schimmel, this study would not have been possible were it not for your time, energy, expertise, and support. I am grateful to have had a wonderful committee who stressed the importance of continued growth and learning. To Vicki, thank you for your kindness, patience, and dedication.

To my family, thank you for your constant encouragement, care, and dedication to helping me pursue my goals. It is because of you that I've had the privilege to commit myself to higher education. To all my friends, thank you for being a part of my life and believing in me. To Mary Kate, who has been with me through all of the ups and downs of this challenging process. I would not have been able to do this without you. I love you. 


\section{Table of Contents}

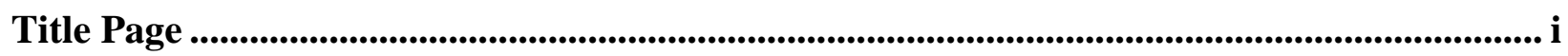

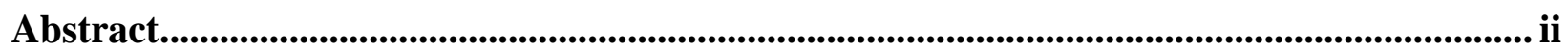

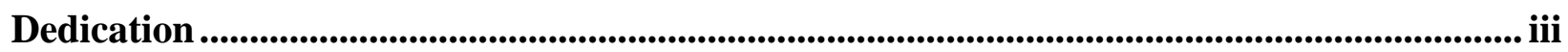

Acknowledgements ....................................................................................................................... iv

Table of Contents ............................................................................................................................................... $\mathrm{v}$

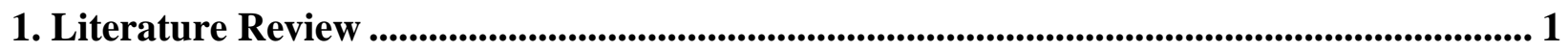

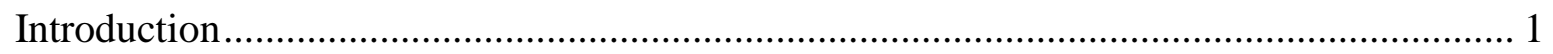

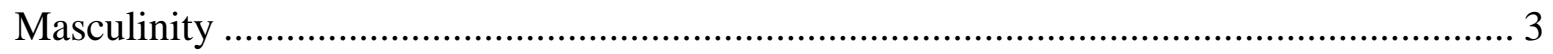

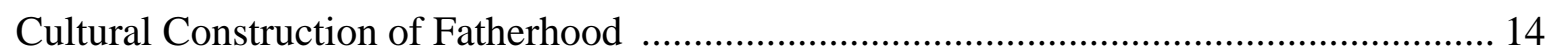

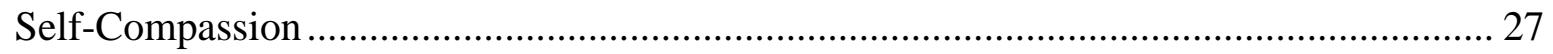

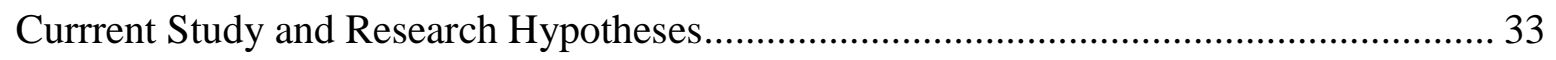

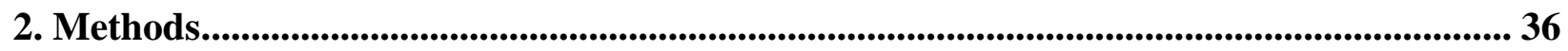

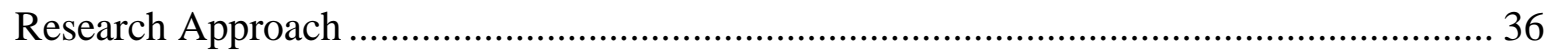

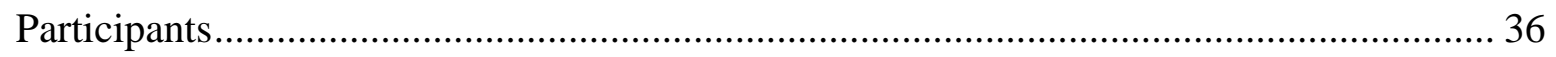

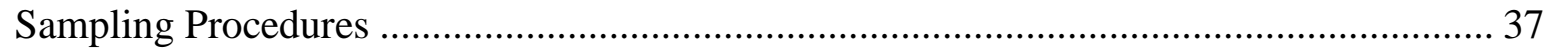

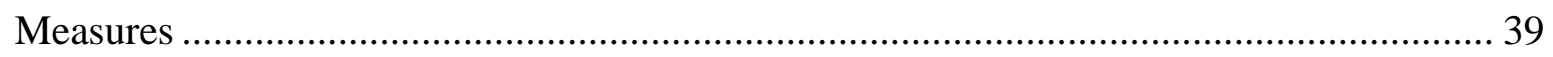

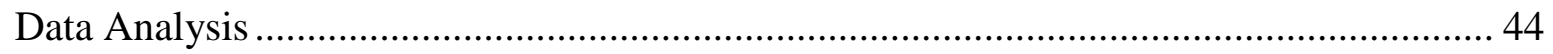

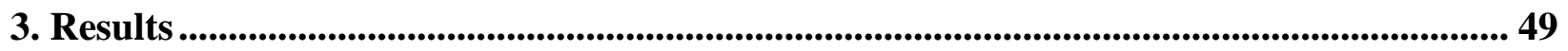

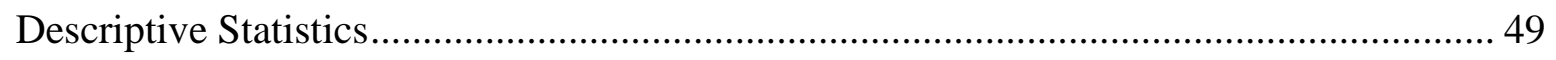

Assumptions and Reliability of Measures ……………................................................... 52

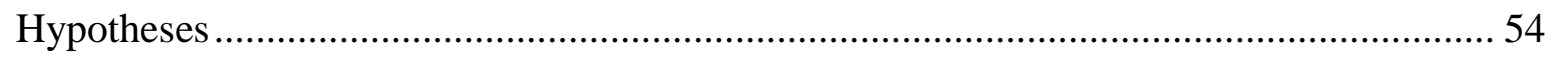

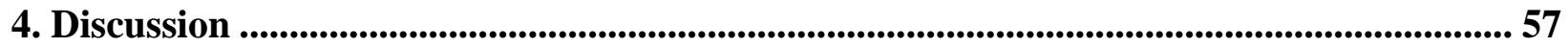




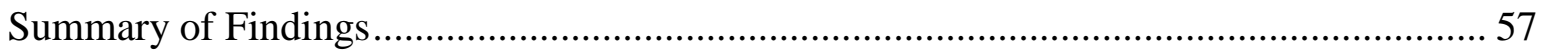

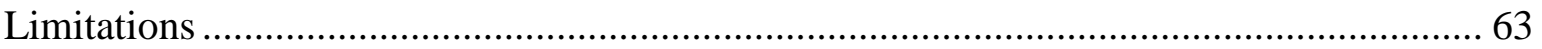

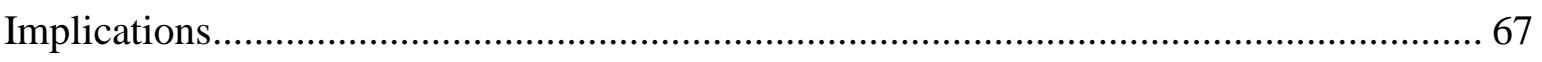

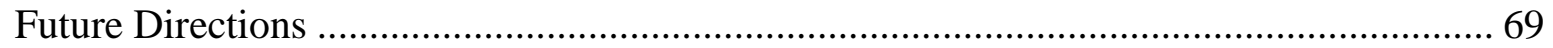

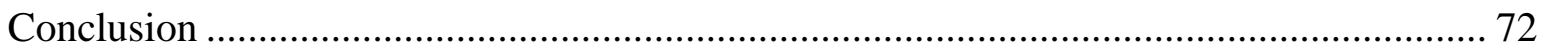

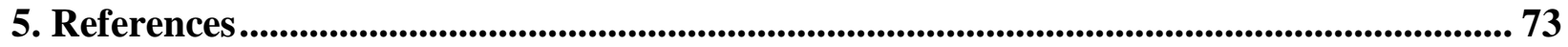

Appendix A

Appendix B ................................................................................................................................ 89

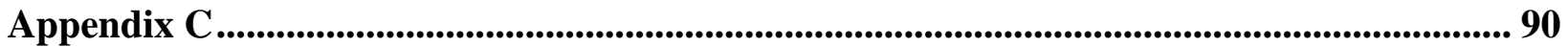

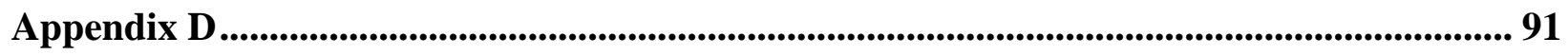

Appendix E

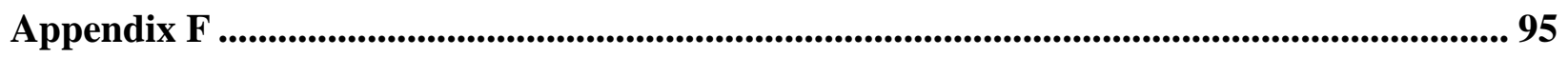

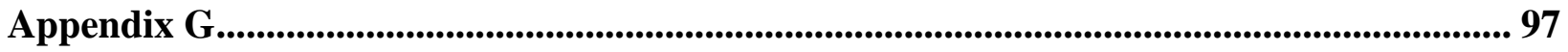

Appendix

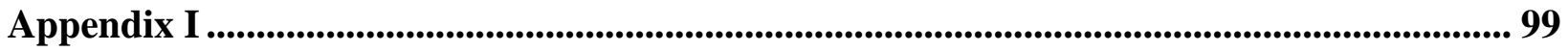




\section{Chapter I: Literature Review}

\section{Introduction}

The socialization processes of men in modern society have been extensively studied. In fact, the American Psychological Association's Division 51 (Society for the Psychological Study of Men and Masculinity) publishes a journal titled the Psychology of Men and Masculinity on a quarterly basis. The current editor of this publication, William Ming Liu, suggested that the growing research on men and masculinity has resulted in a better understanding of the difficulties that men face. He noted several of these difficulties including meeting masculine expectations, stigma surrounding seeking help, concerns related to fatherhood, and how men perceive themselves and the implications these perceptions have in relating to others (Lui, 2016).

In his paper of how constructions of masculinity impact men's well-being, Courtenay (2000) suggested that men must adopt certain unhealthy beliefs and behaviors in order to adhere to culturally normed masculine ideals. Addis and Mahalik (2003), prominent researchers in the area of men and masculinity, commented on the negative impacts of male gender-role socialization processes. These authors purported that there are negative affective, behavioral, and cognitive effects including the preoccupation with power, homophobia, and restricted emotionality, to mention just a few. The effects of gender-role socialization processes on men are further explored in the masculinity section of the literature review.

Neff (2003a), a pioneer within the realm of self-compassion research, stated that all people deserve to be treated with caring and kindness, and in turn, individuals should treat themselves in a similar fashion. The author suggested that individuals could benefit from being more self-compassionate because it might help deter self-critical behaviors, encourage connection with others, and promote dealing with difficult emotional experiences with enhanced 
composure and clarity. Given Liu's (2016) notion that some of the difficulties facing men involve men's perceptions of themselves and the implications for interpersonal relationships, it seems fitting to continue the study of this population's levels of self-compassion and the potential benefits of increased self-compassion.

In an effort to explore levels of self-compassion in individuals, it is important to understand what factors contribute to the development of self-compassion. Early childhood experiences, more specifically, parenting, is a solid place from which to start. Irons, Gilbert, Baldwin, Baccus, and Palmer (2006) found that parenting practices impact how adults later in life engage in self-to-self relating (e.g., being self-reassuring versus self-critical). Therefore, early attachment experiences focusing on parental warmth as opposed to parental rejection may have implications for the development of positive self-relating (e.g., maintaining a selfcompassionate stance).

In addition to parental factors, gender may also be important to study within the context of self-compassion. Yarnell et al. (2015) noted that gender norms have the propensity to obstruct the development of self-compassion in both men and women. While the authors found that men endorsed slightly higher levels of self-compassion compared to women, it may be important to further understand males' within-group differences in regards to self-compassion. Given that gender norms may influence levels of self-compassion, it could be that males' adherence to masculine norms impacts levels of self-compassion.

Men, and society as a whole, can benefit from better understanding the ways in which men are socialized, how they relate to others, and for the purposes of the current study, how they relate to themselves. Liu (2005) indicated that men are socialized to adhere to certain expectations, norms, and values associated with their specific culture. Therefore, the author 
suggested that an understanding of men and masculinity issues from a multicultural perspective could be beneficial. He noted that adherence to masculine norms has negative psychological and relational impacts and that finding more adaptive ways of deconstructing systems of power and oppression can benefit not only men but also women and society as a whole. From a clinical practice standpoint, Liu (2005) also asserted that clinicians working with men could benefit by gaining familiarity with masculine cultural values and deepening awareness of their own biases and assumptions about men. In sum, the current study will seek to better understand how adherence to masculine norms and perceptions of paternal nurturance contribute to levels of selfcompassion in college-aged men.

\section{Masculinity}

Echabe (2010) indicated that in regards to gender, there is disproportionate power when focusing on economic, political, and social factors. Social scientists have recognized that many societies structure and justify their systems of hierarchy. These justifications may look different for any given society. Women are typically placed at the bottom of power hierarchies whereas men are typically at the top (Echabe, 2010). Unfortunately, it can be argued that the American system continues to reflect this notion.

From a historical perspective, men and boys were the primary target of most psychological research. Men were representative of all humanity and masculine norms were the standard for both men and women. This persisted until the 1960's; however, feminist scholars have argued against these perspectives and produced a new and more modern psychology of women. Similarly, men's studies scholars from varying fields generated a new psychology of men with hopes of better understanding masculinity. Researchers from this framework view masculinity as complex and oftentimes problematic, as opposed to the standard of human behavior (Levant, 2011). 
Before further discussing masculinity, it is important to operationalize what the construct entails. Addis, Mansfield, and Syzdek (2010) suggested that masculinity is operationalized in several different ways within current research. The authors indicated that the definition of masculinity is frequently contingent upon responses to questionnaires measuring gender roles, gender ideologies, conformity to gender norms, or related constructs. In general, however, masculinity is often defined as a socially constructed set of gender norms for men (Levant, 2011). A deeper examination of the issues that may arise when men do or do not conform to masculine norms is warranted.

Mankowski and Maton (2010) indicated that the male status has been somewhat paradoxical in that men have both benefitted from and been harmed by their socialization processes. Males do have social and economic power over women as evidenced by their more prominent roles in media, government, and business. White, educated, heterosexual males have predominantly held these positions, thus benefitting most from this historical power structure. However, these authors argued that the various stereotypical behaviors expected from men within their socialization processes have the potential to be damaging (Mankowski \& Maton, 2010).

Conformity to masculine norms. West and Zimmerman (1987) suggested that developing one's gender is “a complex of socially guided perceptual, interactional, and micropolitical activities that cast particular pursuits as expressions of masculine and feminine "natures"' (p. 126). Echabe (2010) proposed that gender identity is possibly the most important identity among all those that compose the global self. Men possess diverse temperaments, physical traits, and personality traits that impact their level of adherence to masculinity norms and ideologies. An individual who strongly identifies with masculine qualities or norms will not 
inevitably encounter negative outcomes. Difficulties do arise when men experience the pressure to modify their behavior and/or beliefs, especially in the service of conformity to masculine standards. Therefore, the resulting distress may be related to how one's masculine identity conflicts with their existing values or belief systems (Chu, 2014).

In his review of the literature, O’Neil (2013) found that gender role conflict significantly correlated with the following mental health-related difficulties across different cultural and racial groups; anxiety, depression, substance use/abuse, low self-esteem, low ego identity, high authoritarianism, negative personality styles, shame, and problems with coping skills. Furthermore, his review identified 39 interpersonal difficulties that have significantly correlated with gender role conflict including, but not limited to, lack of interpersonal closeness, decreased emotional expressiveness, discriminatory behaviors and attitudes, and marital dissatisfaction.

Chu (2014) noted that not all masculine norms are damaging. So, it is important to understand how and when conforming to masculine norms might become problematic. The author used three masculinity related constructs (i.e., independence, toughness, and stoicism) to demonstrate when they can become harmful to males. Independence may become damaging when it goes beyond healthy self-sufficiency and instead leads to feelings of isolation and loneliness. Toughness can become problematic when boys go beyond demonstrating strength and/or power and instead use violent or aggressive means to manipulate others, thus creating a competitive and sometimes hostile environment. When males conform to norms emphasizing stoicism beyond healthy limits, they can become dissociated from their internal experiences as well as disengaged in their relationships. The author therefore concluded that conforming to masculine norms becomes particularly destructive for boys when silences, distortions, and disconnections develop within males. 
In sum, it is rather clear given the literature that masculinity is complex, multifaceted, and sometimes problematic depending upon one's level of adherence to certain masculine norms and stereotypes. A deeper exploration of concepts related to masculinity may further elaborate why it is essential to study male behavior from a societal/cultural perspective.

Distinctiveness of conformity to masculine norms as a construct. Parent, Moradi, Rummell, and Tokar (2011) indicated that previous studies have questioned the distinctiveness of masculinity-related constructs from personality factors and self-esteem. The authors suggested that there have been discriminative validity limitations in previous approaches attempting to operationalize and conceptualize concepts related to masculinity. More specifically, the authors noted that the distinctiveness of conformity to masculine norms from the constructs of personality variables and self-esteem has not been established. Therefore, they sought to establish discriminative validity for the construct.

Parent et al. (2011) found results that supported the discriminant validity of conformity to masculine norms in relation to self-esteem and various personality dimensions. The authors concluded that assessing masculine gender role conformity might provide distinctive information outside the scope of personality and self-esteem assessments.

Parent et al. (2011) distinguished between masculinity-related constructs in their research related to conformity to masculine norms. They particularly dealt with three related but distinctly separate constructs that included gender role ideology, gender role conflict, and conformity to masculine norms. The authors proposed that gender role ideology refers to beliefs about the behaviors that women and men in general should or should not engage in due to their gender. They went on to suggest that gender role conflict involves stress that arises from gender role prescriptions; whereas conformity to masculine norms is different because it is examined within 
the context of dominant U.S. culture and is grounded in the idea that men of diverse backgrounds within the U.S. are held to dominant cultural standards (Parent et al., 2011).

O’Neil (2012) reviewed over 200 studies concerning masculine constructs, including conformity to masculine gender roles. He found that many of these constructs were significantly correlated with men's psychological and interpersonal struggles. In specifically reviewing studies concerning conformity to men's gender roles, the author noted that this construct was significantly correlated with harmful alcohol use, decreased help-seeking involving emotional difficulties, increased physical altercations, difficulties with anger management, increased risktaking behaviors, increased marijuana use, increased binge drinking, decreased health promotion behaviors, increased sexism, internalized homophobia, poorer sexual functioning, racial identities in the pre-encounter phase, lower self-esteem, psychological distress, and masculine body ideal distress (O’Neil, 2012). These findings are alarming and empirically support the idea that men who adhere to more rigid forms of masculinity can experience significant issues related to their well-being.

Gender Role Strain Paradigm and related constructs. Rummell and Levant (2014) studied the gender role strain paradigm (GRSP), which was grounded within social psychology and is correlated with terms including conformity, social cognition theory, stereotypes, norms, and gender roles. This paradigm focuses on gender roles as constructed from norms and stereotypes that evolve from dominant gender ideologies in society at large. Boys and men perceive societal pressure to conform to gender role norms through social interactions from which they are punished, reinforced, and are provided a vast array of opportunities for vicarious learning. Overall, these norms can be incongruent and contradictory. Therefore, they may be 
frequently violated. These violations can result in social disapproval and distressing psychological outcomes (Rummel \& Levant, 2014).

The male socialization process requires men to demonstrate signs and behaviors of selfreliance, independence, and toughness. Men are supposed to be oriented towards achievement, emotional constriction, and competitiveness. However, these traits are sometimes maladaptive and unhealthy. Research has shown that males who do endorse such characteristics do not tend to utilize psychological services and are typically unaware of their own intrapersonal levels of distress or symptoms of ill health (Nguyen, Ming Liu, Hernandez, \& Stinson, 2012). It may be important to further examine how gender role socialization and masculinity impact mental health and help-seeking behaviors.

O’Neil (2013) suggested that gender role conflict limits individuals to stereotypical and rigid norms of masculinity ideology, which Thompson and Peck (1995) suggested is the core construct in the body of research assessing attitudes toward men and male roles. These limitations are referred to as gender role restrictions. Such restrictions cause people to conform to gender norms that may result in limiting others' potential and decrease freedom. O'Neil (2013) further indicated that men self-restrict in a variety of ways which limits their adaptability and behavioral flexibility.

Specifically, O’Neil (2013) suggested that men restrict themselves by dedicating their energy to work while allowing their family and parenting roles to serve as a secondary role. This may ultimately decrease opportunities for intimacy, promote burnout, and decrease self-care behaviors. Gender role constriction also involves forcing inflexible gender roles on others through forced conformity to feminine or masculine ideology, which can include emotional abuse, manipulation, criticism, and excessive control (O’Neil, 2013). 
O’Neil (2013) also described the constructs of gender role devaluation and gender violations. He defined gender role devaluations as "negative critiques of self or others when conforming to, deviating from, or violating stereotypical gender role norms of masculinity ideology" (p. 493). He explained that these devaluations result in reducing self-esteem, status, or stature. Men may devalue themselves when they fail to achieve the masculine norms prescribed by masculine ideologies. The described gender role violations are the most extreme type of gender role conflict. These involve causing direct harm to the self or others due to destructive gender role norms of masculinity ideology. Reidy, Shirk, Sloan, and Zeichner (2009) indicated that men who more strongly adhere to traditional male gender norms may be more likely to be perpetrators of violence, specifically against women who are perceived to violate traditional gender role norms.

Examining the construct of gender norm violations more thoroughly, it appears that some males can also be uncomfortable with females who adhere to typical feminine norms or stereotypes. O’Neil (2013) defined the fear of femininity as negative emotions and thoughts connected to stereotypical feminine behaviors, values, and attitudes. He suggested that these fears are learned early in childhood while one's gender role identity is being influenced by parents, peers, and society as a whole.

Masculine conformity and culture. Levant and Richmond (2007) reviewed the masculinity ideologies research and found that men endorse traditional masculinity ideology more than women. Also, most of the studies they focused on in their review demonstrated that sex had a larger effect size than either race or ethnicity. The authors also found that African Americans endorse traditional masculinity ideology to a greater extent than European Americans, whereas the Latina/Latinos in the United States and Puerto Rico fall somewhere in 
the middle between European Americans and African Americans in regards to endorsement. Furthermore, the effects of race on African Americans' endorsement of traditional masculinity ideology was moderated by geographic residence, whereas for seven separate cultural groups of Asian American men, no differences were found in the endorsement of traditional masculinity. The authors also noted that individuals identified in the lower social class have a greater likelihood of endorsing traditional masculinity ideology.

In further support of these findings, Gordon et al. (2013) examined adherence to traditional masculine norms in a sample of 296 racially and ethnically diverse men transitioning to fatherhood. The authors found that African American men adhered to stricter masculine ideologies than the Latino and White males included in the study.

Mental health and masculinity. Compared to women, men are not as likely to seek help for a variety of health concerns (Sierra Hernandez, Han, Oliffe, \& Ogrodniczuk, 2014). Seeking psychotherapeutic help for men typically sets the stage for gender role conflict. Men who do seek counseling often find that this contradicts their beliefs, especially the beliefs of independence and self-reliance due to men thinking that attending counseling means they are unable to solve their own problems. Furthermore, when actually in therapy, the process usually requires men to experience and express their emotions. The process also requires vulnerability. Therefore, mental health services may be disagreeable for some men (Nguyen et al., 2012).

Yousaf, Popat, and Hunter (2014) indicated that men endorse more reluctant attitudes towards seeking help for mental health and psychological difficulties. Related literature has suggested that these attitudes may be correlated with men's level of adherence to masculinity norms. Moreover, male gender roles promoting stoicism, avoidance of weakness, and selfreliance may increase self-stigmatizing perceptions. Consequently, these difficulties may lead to 
more negative attitudes towards counseling in general (Sierra Hernandez et al., 2014). Heath, Brenner, Vogel, Lannin, and Strass (2017) found that adherence to masculine norms was associated with increased help-seeking self-disclosure risks and self-stigma.

Sierra Hernandez et al. (2014) proposed that men's mental health help-seeking attitudes were predicted by attitudes related to traditional masculinity norms. Therefore, the authors indicated that examining men's attitudes about masculinity might be the best approach in addressing their low rates of help-seeking. However, it may be important to identify exactly towards which specific factors of psychological help men hold negative attitudes (Berger, Addis, Green, Mackowiak, \& Goldberg, 2013). These authors purported that men held more favorable attitudes towards receiving psychotherapy compared to various other forms of help-seeking, including medication therapy. Despite their findings that men's attitudes towards help-seeking were predominantly ambivalent overall, they suggest that men may not always be resistant to psychological help; instead, they may hold variable levels of ambivalence contingent upon the nature of help (Berger et al., 2013).

When considering men's attitudes towards specific forms of help-seeking, it is also important to be cognizant of the numerous multicultural variables that may impact the research. Vogel, Heimerdinger-Edwards, Hammer, and Hubbard (2011) indicated that emotional expressivity, mental health literacy, acculturation, multiple stigmas, self-concealment, and individual versus collective orientations can influence men's help-seeking behaviors. Therefore, further studying men's attitudes of various forms of help-seeking while accounting for levels of adherence to masculine norms and cultural variables may be an important addition to the current literature in masculinity. In addition to men's help-seeking behaviors, it may be important to 
understand the stereotypes and gender differences involved in mental health and mental disorders.

Men, stereotypes, and mental illness. Boysen, Ebersole, Casner, and Coston (2014) suggested that mental illness is stigmatized and that there are differences in the levels of stigmatization depending on the given mental disorder. The authors contended that there is an intersection between stereotypes about gender and mental disorders as well that may impact the way society views mental illness. Furthermore, these authors found that the perceived masculinity or femininity of mental disorders predicted levels of stigmatization. Masculine disorders were found to produce increased levels of stigmatization as measured by a broad range of attitudes (Boysen et al., 2014).

Boysen et al. (2014) concluded that mental disorders are gendered. They found that masculine stereotyped disorders were characterized by externalization. These disorders included antisocial personality disorder and substance use disorders. Even more common were disorders associated with abnormal sexual interests. In contrast, the feminine stereotype was associated with concerns about body image, sexual dysfunction, and emotionality. Men appear to be understood as more aggressive and sexually inappropriate whereas women are seen as more emotional and concerned with their physical appearance. Moreover, this complex interaction of gender of mental illness may be an important factor in examining stigmatization (Boysen et al., 2014).

There is some empirical evidence demonstrating the impact that conformity to masculine norms has on men's behavior, socialization processes, mental health, and even their help-seeking behaviors (Chu, 2014; Nguyen et al., 2012; O’Neil, 2013; Reidy et al., 2009; Sierra Hernandez et al., 2014; Vogel et al., 2011; Yousaf et al., 2014). Whereas it appears most of these effects can 
be negative depending upon the rigidity of adherence, much of the literature on self-compassion seems to point to the opposite and provides evidence for the positive benefits of greater levels of self-compassion. It is therefore important to examine psychological constructs (e.g., selfcompassion) that could be helpful to this population.

Intersection of self-compassion and masculinity. Previous research has shown that men with high levels of conformity to masculine norms may have more difficulty managing vulnerable intrapersonal emotional states (Reilly, Rochlen, \& Awad, 2014). The authors suggested that it may be difficult to maintain a self-compassionate perspective while simultaneously adhering to masculine norms, as these two constructs may be incongruous. More specifically, the authors proposed that high levels of adherence to masculine norms paired with high levels of trait shame might decrease men's predilection for self-compassion. However, this may not have the same effect on men's self-esteem. Therefore, the authors noted that adherence to masculine behavior may actually be associated with higher self-esteem, especially for those who experience shame (Reilly et al., 2014).

Reilly et al. (2014) further found that men's feelings of emotional restrictiveness, inadequacy, and self-criticalness were discordant with levels of self-compassion. The authors stated that overall, self-compassion was negatively correlated with conformity to masculine norms, thus demonstrating the importance of gender orientation and self-compassion. Ultimately, the idea of self-compassion may be difficult for men who strongly adhere to masculine norms and/or maintain high levels of trait shame. Interestingly, Heath et al. (2017) found that selfcompassion can serve as a buffer between adherence to masculine norms and the difficulties men experience associated with seeking help (i.e., self-stigma and self-disclosure risks). The authors noted that college-aged men who evidence high levels of self-compassion may have the capacity 
to adhere to masculine norms without feeling ashamed or self-critical when engaging in behaviors not typically considered masculine (e.g., seeking help for mental health concerns).

Lockard, Hayes, Neff, and Locke (2014) indicated that there has not been much research examining the relationship between levels of self-compassion and gender in college students. In sum, I suggest that more research is needed to further identify the relationship between adherence to masculinity norms and levels of self-compassion. The current study will examine this relationship further.

\section{Cultural Construction of Fatherhood}

The family continues to be viewed as the major contributor to children's socialization. This includes the development of children's social skills, values, social orientations, and even personality attributes. Furthermore, the affective components of parent-child relationships are of continued interest as they may play a central role in children's socialization (Maccoby, 1992). Therefore, it may be important to further investigate the relationships among the father-son dyad, self-compassion, and adherence to masculinity. Before exploring these possible connections, it will be necessary to expand upon the construct of fatherhood.

According to the United States Census Bureau (2014), there was an estimated 70.1 million fathers in the U.S. with 24.7 million of those fathers being part of a married-couple family with children younger than 18-years-old in 2013. It was also estimated that there were 2 million single fathers as of 2013 (U.S. Census Bureau, 2014). Rohner and Veneziano (2001) suggested that fatherhood is culturally constructed, and, as a construct, it is impacted by cultural understandings of masculinity and femininity. They contended that popular literature contains many references to mothering and parenting; however, fathering is rarely used in these contexts. The authors noted that beliefs about fathers prior to the 1970's encompassed the notion that 
fathers are frequently incompetent and perhaps biologically unfit for the responsibilities of child rearing. Also, the influence of fathers on children's development was seen as predominantly inconsequential or at least indirect. Conversely, mothers have been seen as biologically programmed to be caregivers as they can provide all the essentials necessary for healthy childhood development. The authors shared that these views have persisted until the time they wrote their article in 2001.

Unfortunately, Rohner and Veneziano (2001) also shared that these beliefs have influenced the amount of research encompassing fatherhood and the influence fathers have on the development of their children, leaving more interest in studying the effects of motherhood. It wasn't until the 1960's and 1970's that research on fatherhood more significantly emerged within childhood development and family studies. Within this time frame, an increase in fathering research began to emerge due to surmounting evidence for the importance of fathering as well as the feminist movement that led to a reexamination of the masculine gender identity. Another major influence on fathering research was the rise of effective multivariate statistical packages with the ability to perform multiple regressions and structural equation modeling. These analyses helped to show that father love explained independent portions of certain positive outcomes beyond that of mother love, thus providing further support for the importance of a nurturing and caring father (Rohner \& Veneziano, 2001).

Paternal involvement and masculinity. Chu (2014) indicated that early efforts aimed at challenging harmful effects of boys' socialization have focused on modifying the messages that boys receive while also mitigating the pressure that boys feel to conform to group norms of masculinity. However, boys play an active role in their own socialization processes, and they can alleviate some of the harmful effects impacting their learning and development. The author 
further suggested that in addition to considering the contexts and content of boys' socialization, exploring relational and psychological factors is also necessary in examining the influence of socialization processes. Therefore, it may be important to understand how males' relationships with their parents may impact subsequent functioning.

Lewis and Lamb (2003) suggested that previous researchers have demonstrated that mothers have superior skills when interacting with their children and maternal closeness has a more significant effect on children. The authors noted that historically, fathers may have been less available to interact, thus decreasing the amount of time spent caring for children. However, research has also shown that fathers' involvement with their children is associated with better outcomes for children as well as family functioning (Glass \& Owen, 2010). For example, positive paternal involvement has been found to be positively and significantly correlated with adolescent resiliency (Zhang, Zhao, Ju, \& Ma, 2015), greater self-esteem in children (Flouri, 2004), and greater marital satisfaction and maternal psychological functioning (Hansen, Weissbrod, Schwartz, \& Taylor, 2012).

Bonney, Kelley, and Levant (1999) suggested that paternal involvement in childcare is more self-determined than previously believed. In their study, they found that fathers who reported a liberal gender role ideology had more progressive views towards fathering. These progressive views of fathering were related to fathers' and mothers' reports of fathers' involvement in caregiving behaviors. Therefore, the authors indicated that beliefs about masculinity related to both attitudes of fathering as well as fathers' involvement in childcare. These findings underscore the importance of fathers' gender role ideology in caregiver involvement. 
Fathers who hold more liberal views about gender role ideology have been found to endorse increased involvement in child care behaviors and more time spent as child's primary care giver (Bonney, Kelley, \& Levant, 1999). However, the authors noted that spending time as the primary caregiver is influenced by potential structural barriers, specifically, one's job schedule. Their results indicated that fathers who spent more hours at their job spent less time in the role of primary caregiver and were ultimately less likely to engage in childcare activities. However, they did suggest that fathers might demonstrate paternal involvement through providing for their families via working. Therefore, a father who is less involved in direct childcare activities may also feel a high level of family responsibility. But this can be problematic given that research has demonstrated less paternal involvement is associated with negative short-term and long-term effects on children's health, education, and psychosocial development (Dearden et. al., 2013).

In their review, Levant and Richmond (2007) found generational differences related to traditional masculinity ideology. Specifically, they suggested that past studies have found that sons were less likely to adhere to traditional male gender role norms compared to their fathers. In addition, traditional masculinity ideology has been associated with problematic individual and relational variables, including lower paternal participation in childcare and more negative paternal beliefs about the father's role.

In sum, fathers do have an important role in children's socialization processes. It appears that paternal involvement can positively benefit children, however, this may depend upon the quality of the relationship. There seem to be several variables that may impact the quality of the relationship, including issues related to adherence to traditional masculinity. It may be necessary 
to take a more in-depth look at how paternal love and acceptance may further impact human development.

Impact of father love. Rohner and Veneziano (2001) reported that paternal love and acceptance has been increasingly implicated in both children's and adults' psychological health and well-being across the lifespan. The authors shared that paternal and maternal love and acceptance have been shown to be related to overall sense of well-being and happiness, psychological health, physical health, academic achievement, social competence, and the internalization of parental values. Conversely, maternal and paternal withdrawal/rejection has been shown to have significant effects on self-concept, self-esteem, emotional/social withdrawal, anxiety, depression, conduct issues, substance use, emotional instability, and cognitive/academic issues.

Therefore, the positive outcomes of paternal love and acceptance do have empirical support. It may be worthwhile to explore some different types of fathering roles to further understand the perceptions of children about their relationships with their fathers and the potential impacts of these perceptions.

Expressive versus instrumental fathering functions. Finley and Schwartz (2006) found that young adult children from both divorced and intact families reported that the highest levels of paternal involvement were in instrumental functions (e.g., providing protection, discipline, providing financial support) as opposed to expressive functions (e.g., sharing activities, caregiving, companionship). The authors noted that this supports the notion that fathers continue to engage in more instrumental fathering roles versus expressive fathering roles.

However, fathers from intact families in all eight of the ethnic groups studied (i.e., nonHispanic White, non-Cuban Hispanic, U.S.-born Cuban, Caribbean Islander, foreign-born 
Cuban, Asian, mixed ethnicity, and African American) were endorsed as being somewhat involved in expressive functions. Therefore, the authors concluded that fathers from intact families demonstrated some degree of expressive fathering. In examining fathers from divorced families, the authors reported that levels of expressive and instrumental fathering were lower (Finley \& Schwartz, 2006).

Finley and Schwartz (2006) noted that the variable of ethnicity only moderated the magnitude of the difference between instrumental and expressive fathering mean scores. Furthermore, they found no gender differences among the children. The authors concluded that their results provided support for cross-ethnic and cross-gender consistency.

Finley and Schwartz (2006) compared their findings to Parsons and Bales's (1955) classic theoretical work. Finley and Schwartz (2006) concluded that their findings supported Parsons and Bales's (1955) conceptualization of fathering in that instrumental functions continue to be associated with the primary roles of fatherhood. The authors noted that the traditional instrumental functions (e.g., protection, facilitating moral/ethical development, discipline, providing income, and fostering the development of responsibility) continue to be most salient in fathering. The authors shared that despite recent efforts to stimulate enhanced nurturance and expressive involvement in fathering practice, an apparent disparity still exists between fathers' involvement in expressive versus instrumental roles.

Therefore, it seems that fathers are viewed as less likely to engage in the nurturing behaviors more typically associated with expressive fathering functions. This could be problematic given that parental love and acceptance is associated with more positive outcomes (Rohner \& Veneziano, 2001). It could be hypothesized that expressive functions are perceived as more nurturing whereas instrumental functions (e.g., providing discipline, instilling morals) may 
be less so. However, it may be that instrumental functions serve as another form of love and/or acceptance. Also, perceptions of paternal warmth and/or love may be further impacted by gender role ideologies and adherence to masculine norms. A further exploration of the impact of paternal nurturance is warranted.

Relationship between nurturance and involvement. Doyle, Pecukonis, and Lindsey (2015) examined the impact of paternal nurturance on the psychological well-being of African American youth. The authors found that their participants endorsed greater levels of perceived paternal nurturance given the frequency and duration of time spent interacting with their fathers from the ages of birth to 18 years old. In addition, the authors noted that their statistical analysis supported the separateness and interrelatedness of paternal nurturance and paternal involvement as two distinct constructs. They shared that fathers' presence impacted the youths' perceptions of nurturance. Therefore, the amount of time spent together influenced how these youths felt in regard to how nurturing their fathers were.

One surprising finding was that levels of perceived paternal nurturance were not significantly affected by fathers' residential status. The authors concluded that residential status may be a complex variable given that some residential fathers spent less time with their children, whereas there may be some nonresidential fathers who spend a great deal of time with their children. In addition, father type (i.e., biological versus other) was not significant within the regression analysis (Doyle, Pecukonis, \& Lindsey, 2015).

Doyle et al. (2015) also found that perceptions of paternal nurturance were not a significant predictor of youth psychological well-being. However, they did find weak correlations between paternal nurturance and instability and unresponsiveness - two emotional components of psychological well-being. They noted that these findings contradicted previous 
studies on the relationship between youth well-being and perceptions of paternal nurturance. The authors noted that this may be because they used the Nurturant Fathering Scale (NFS) instead of the Parental Acceptance-Rejection Questionnaire (PARQ). They further reported that these inconsistencies may be due to sampling differences as well. Overall, it is unclear as to what may have impacted these results. Therefore, further studies regarding perceptions of paternal nurturance and well-being are needed to further understand how the dyadic relationship impacts future well-being and functioning. Moving along the developmental spectrum, it is critical to explore the impact that fathers have on emerging adults.

Fathers' impact on emerging adults. Schwartz et al. (2009) stated that fathers might have a greater impact on the outcomes of emerging adults as opposed to adolescents. In their sample of 1,546 college students, Schwartz et al. (2009) found that perceptions of parental acceptance when the participants were adolescents, specifically paternal acceptance, was strongly protective against numerous health-risk behaviors. These health-risk behaviors included substance use, casual sex, operating a vehicle while under the influence of drugs or alcohol, and riding as a passenger with someone who had been using illicit substances or alcohol. The authors noted that the protective effects were stronger for the low-frequency and more dangerous behaviors such as hard drug use, impaired driving, casual sex, and the misuse of prescription drugs. The effects were less strong for the more normative behaviors including oral sex, binge drinking, and marijuana use. It is important to note that perceived acceptance from fathers made a greater contribution to the behaviors examined beyond that of maternal acceptance.

The authors provided several conclusions based upon their results. They indicated that retrospective accounts of perceived fathering might have long-term impacts on adult children's lives and health-risk behaviors. Furthermore, these results were consistent across four ethnic 
groups (i.e., Asian, Hispanic, Black, and White) and whether the participants' parents were living together or not as they were growing up. In this study, $80 \%$ of the divorced fathers were mainly nonresidential and had a comparable level of influence on their children's health-risk behaviors (Schwartz et al., 2009).

In addition, the authors concluded that support, guidance, and nurturance provided by the parents could help individuals transition to adulthood - and fathers may play a greater role in their children's health-risk behaviors. They purported that more research is needed to understand the effects of perceived parenting on other outcomes in emerging adulthood (Schwartz et al., 2009).

It is apparent that paternal nurturance can serve as a protective factor against various health-risk behaviors and negative outcomes. The literature appears to support this finding regardless of ethnicity and whether or not parents reside together. These findings provide important implications regarding family factors and perceptions of paternal nurturance. Further exploration between various family structures and paternal nurturance is warranted.

Doyle et al. (2015) noted the importance of children having frequent contact with their fathers and their perceptions of paternal nurturance. Schwartz and Finley (2005) found that both reported levels of paternal nurturance and paternal involvement were significantly lower for participants from divorced families versus participants from intact families. This finding was consistent across all ethnic groups (i.e., non-Hispanic White, non-Hispanic Black, Hispanic, Asian, and 4\% mixed ethnicity). Overall, family form differences (i.e., intact families versus divorced families) in paternal involvement and nurturance were most pronounced for African Americans, mixed-ethnicity individuals, foreign-born Cubans, and Caribbean Islanders. Non- 
Hispanic Whites and Asians endorsed the smallest differences. Moderate differences were seen in U.S.-born Cubans and non-Cuban Hispanics.

In continuing their exploration of paternal nurturance while appreciating diversity variables, Schwartz and Finley (2006) noted the importance of examining the impact of fathering by nonbiological fathers in nontraditional families (i.e., stepfamilies, adoptive families, and adoptive stepfamilies). In their own study, the authors looked at the relationships of paternal nurturance, father involvement, and desired father involvement to young adult psychosocial functioning. They found that expressive father involvement and perceptions of paternal nurturance were positively and strongly associated with current psychosocial functioning for young adults from adoptive families. The results were significantly stronger compared to the young adults coming from adoptive and nonadoptive stepfamilies. The mentoring/advising aspects of paternal involvement were less strongly associated with psychosocial functioning. The young adults in the study did not evidence any differences between those coming from adoptive stepfathers and nonadoptive stepfamilies on any of the paternal measures.

Schwartz and Finley (2006) found that the age of the child at which their father entered their lives and the amount of time the father was involved served as moderating variables. The relationship between the number of years of father involvement and reported fathering variables were positively and strongly related in adoptive stepfamilies and nearly zero in nonadoptive stepfamilies. In conclusion, the authors suggested that the correlations of earlier age at the time of stepfather entry and more years of involvement with increased positive ratings of fathering could reflect the commitment of adoptive stepfathers. The opposite was found for nonadoptive stepfamilies, and requires further study (Schwartz \& Finley, 2006). Overall, nonadoptive stepfathers were associated with the lowest levels of paternal nurturance and involvement. 
Therefore, it does appear that there are important differences in perceptions of paternal nurturance depending upon family structure as well as ethnicity in some instances. In addition to these family structures mentioned, it may be important to briefly address how perceptions of paternal nurturance may be impacted by the dyadic marital relationship and vice versa.

Galovan, Holmes, Schramm, and Lee (2014) investigated both the quantity and quality of wives' perceptions of their husbands' relationships with their children. They found that these perceptions were significant predictors of marital quality. The authors concluded that better marital quality may be one outcome of nurturing father-child relationships. Overall, they suggested that these perceptions of paternal nurturance might contribute to not only increased marital quality, but the effects might extend to other areas of family life. Therefore, there are many reasons as to why it is critical to expand upon the study of perceptions of paternal nurturance.

Rohner and Veneziano (2001) stated that the impact of paternal behavior on children has been largely ignored:

Research questions that are regarded as appropriate or sensible at a particular point in time are usually situated within a matrix of cultural beliefs often widely accepted within the dominant population at large, but certainly within the scientific community more specifically. The issue of fatherhood is a case in point. (p. 384)

Rohner and Veneziano (2001) shared that increased research on the importance of father love is needed in order to lessen the frequency of mother-blaming while seeking to increase fathers' motivation for providing nurturance. Doyle et al. (2015) purported that future research should focus on the determinants of youths' psychological well-being and the importance of fathering. The authors noted that certain aspects of fathering may be associated with 
psychological well-being. More specifically, the authors shared that paternal nurturance may be associated with various youth outcomes related to mental health. Furthermore, Doyle et al. (2015) suggested that clinicians should seek to enhance the quality of father-child relationships by encouraging increased and consistent involvement of fathers. In sum, further paternal nurturance research is needed. Self-compassion, as previously explored, may also be an important variable that can be further explored in relation to paternal nurturance. There has been some research done in this area thus far, and it may be important to understand how attachment and the parent-child relationship is implicated in the development of self-compassion.

Parents' impact on self-compassion. Pepping, Davis, O’Donovan, and Pal (2015) noted that individuals can become less efficient at understanding their own need for self-compassion if there is an inconsistency in receiving care and compassion from others. They noted that individuals begin seeking this type of support during childhood, and therefore differences in individuals' levels of self-compassion may be a function of early childhood interactions with caregivers. The authors further shared that the association between the type of parenting received and subsequent levels of self-compassion may be indirect and complex. This could allude to the idea that there may be other factors influencing the relationship between parenting received and self-compassion.

Pepping et al. (2015) examined how early attachment patterns may influence individuals' levels of self-compassion later in life. The authors found that self-soothing abilities may provide an individual with greater capacity for self-compassion, whereas individuals who perceived their parents as rejecting, critical, and lacking in warmth endorsed lower levels of self-compassion due to increased attachment anxiety. This research is further supported by Potter, Keong, Francis, 
and Schuster's (2014) finding that parental criticism was negatively correlated with overall selfcompassion in their sample 211 males and females.

In a related line of research on attachment and self-compassion, Wei, Liao, $\mathrm{Ku}$, and Shaffer (2011) examined self-compassion as a potential mediator between attachment and wellbeing. Their findings showed self-compassion functioned as a mediating variable and explained the negative association between subjective well-being and attachment anxiety. They purported that people who are securely attached may have a greater capacity for self-compassion. From a broader family systems perspective, Neff and McGehee (2010) found that adolescents from closer and more harmonious families endorsed higher levels of self-compassion, whereas adolescents from turbulent and stressful homes were less self-compassionate. Therefore, it appears that the quality of early attachment relationships may have an impact on individuals' levels of self-compassion later in life.

A study conducted by Kelly and Dupasquier (2016) provides further evidence for the importance of quality parent-child relationships in regard to the development of self-compassion. The authors studied a sample of undergraduate female students regarding recalled parental warmth and the capacity for self-compassion. The authors stated that their findings suggested having emotionally warm parents can increase feelings of connectedness, reassurance, and safeness in subsequent adult relationships. They further suggested that having these qualities in adult relationships may facilitate self-compassion because of individuals' ability to turn these feelings inward via the self-soothing system. In their results, they found that recalled parental emotional warmth was related to enhanced capacities for self-compassion and receiving compassion indirectly via feelings of social safeness. Conversely, the authors suggested that individuals who have difficulty feeling connected, reassured, and accepted in their relationships 
due to lack of parental warmth have greater difficulty taking a self-compassionate stance. Those who were raised in less secure family environments may experience self-compassion as threatening, foreign, and/or frightening. Overall, the authors shared that if other-to-self relating lacked warmth, then self-to-self relating may also be deprived of warmth. Therefore, it appears that parents demonstrating warmth and the development of secure attachments are related to increased levels of self-compassion.

\section{Self-Compassion}

Western psychologists have started to develop an empirical and theoretical understanding of self-compassion (Barnard \& Curry, 2011). The construct of self-compassion involves three distinct yet integrated components (Neff, 2003a). These components are interconnected and include self-kindness, a sense of common humanity, and mindfulness (Smeets, Neff, Alberts, \& Peters, 2014). Self-kindness entails being kind and understanding instead of maintaining a selfcritical stance toward oneself (Neff, 2003a). Even after experiencing failure, self-kindness involves taking a stance towards the self that affirms one deserves happiness, affection, and love (Barnard \& Curry, 2011). Self-kindness is in direct opposition to being self-judgmental. Furthermore, it is akin to the Rogerian notion of treating oneself with warmth and unconditional acceptance (Smeets et al., 2014). Lockard, Hayes, Neff, and Locke (2014) added that selfkindness involves self-soothing and the tendency to engage in understanding and caring towards one's self versus being judgmental and/or self-critical.

The second component of self-compassion is having a sense of common humanity. From this perspective, individuals perceive their own experiences as part of the universal human experience versus viewing them as isolating and separating (Neff, 2003a). This idea pertains to understanding and recognizing that imperfections, failures, and making mistakes are inherent to human nature. Having the perspective that all humans have difficulties and deficiencies helps the 
individual to connect with the shared human condition (Lockard et al., 2014). For example, when people are in times of pain or frustration they tend to feel isolated from others-they may withdraw from others and deal with their inadequacies and failures alone (Barnard \& Curry, 2011). Experiencing a sense of common humanity may act as a buffer against such potentially painful feelings.

Mindfulness is the third component of self-compassion (Lockard et al., 2014). The concept of mindfulness has been derived from ancient Buddhist, Hindu, and Chinese traditions. Europe and the United States have utilized modern adaptations of these medical and contemplative traditions. Typically, the strategies now associated with mindfulness are comprised of being in a state of heightened and restful awareness, increased acceptance of direct experience, and decreased ruminations as well as mental judgments (Tang \& Posner, 2013). This concept is associated with being aware of one's present moment experience. When one is being mindful, he or she is balanced and clear-minded in the present moment without engaging in exaggeration or over-identification with the distressing factors of one's life. Neff (2003a) shared that individuals who are mindful hold their distressing thoughts and feelings in balanced awareness. For the purposes of this current study, I will be using Neff's (2003a) three-factor model of self-compassion given the abundance of empirical support and continued lines of research.

Relationships among components of self-compassion. When discussing the components of self-kindness, mindfulness, and common humanity, Neff (2003a) suggested "While these aspects of self-compassion are conceptually distinct, and are experienced differently at the phenomenological level, they also interact so as to mutually enhance and engender one another" (p. 89). She noted that mindfulness is needed in order to appropriately 
gain enough mental space to be able to offer oneself self-kindness while also understanding the universal nature of human experience. The author further suggested that self-kindness and a common sense of humanity can also influence mindfulness (Neff 2003a).

Despite the aforementioned connections, some researchers have disagreed with Neff's views about the relationships among the three constructs comprising self-compassion. Barnard and Curry (2011) indicated that there is little known about the relations among the components making up self-compassion. The authors questioned whether Neff perceived the components as definitively and inherently related or as factors that are positively associated or propagate one another. Therefore, the authors suggested that it might be difficult to discuss any of the components separately from one another. The authors did hypothesize that self-kindness may foster common humanity. Also, common humanity may foster self-kindness and mindfulness. Lastly, mindfulness may foster self-kindness and common humanity. In sum, these components of self-compassion may beget one another, and the absence of one may impact the development of the other two factors (Barnard \& Curry, 2011). Further research is necessary in order to gain a more comprehensive understanding of how the components of self-compassion interrelate and influence one another. In addition to further understanding how these components are related, it is important to discuss what other variables have been studied in relation to self-compassion.

Correlates of self-compassion. There has been increased interest in the potential benefits of self-compassion (Lockard et al., 2014). Barnard and Curry (2011) stated that self-compassion is correlated to positive outcomes in a range of different domains including social connections, achievement, cognitive patterns, and affect. While many of the studies examined were correlational and relied on self-report, the authors nonetheless suggested the literature 
demonstrates preliminary evidence for the use of interventions that target self-compassion for effecting positive change.

Leary, Tate, Adams, Batts Allen, and Hancock (2007) found that self-compassion moderated individuals' responses to negative events that involved embarrassment, rejection, and failure. Those with greater self-compassion experienced lower levels of distressing emotions when exposed to actual, imagined, and remembered events. Furthermore, the individuals who endorsed more self-compassion also accepted more responsibility for negative events. Lastly, the authors found that self-compassionate people were more willing to accept the unfavorable facets of their behavior and character compared to those who were lower in self-compassion. Therefore, it appears that higher levels of self-compassion may act as a buffer against the impact of negative experiences and subjective feelings of distress. It may also be important to understand how self-compassion has been studied in relation to more prolonged feelings of distress as well.

Wong and Mak (2013) utilized a hierarchical regression analysis to examine the relationship between depression and self-compassion. The authors found that all three of the components of self-compassion (i.e., self-kindness, sense of common humanity, and mindfulness) were negatively correlated with depression. Furthermore, the components of selfcompassion differentially moderated the connection between depression and cognitivepersonality vulnerability. Given that self-compassion appears to be beneficial in regard to mental health and well-being, it could be important to explore the positive impact of increasing selfcompassion in individuals in psychotherapeutic settings.

Men, self-compassion, and relationships. Baker and McNulty (2011) proposed that self-compassion may actually be detrimental to individuals' motivation to rectify past mistakes 
and halt their reoccurrence. These authors suggested that self-compassion may either harm or benefit relationships depending on various sources of motivation to correct mistakes in interpersonal relationships. More specifically, the interaction between self-compassion and conscientiousness may predict relationship outcomes.

Baker and McNulty (2011) found that men with both high levels of self-compassion and high levels of conscientiousness had greater motivation to correct interpersonal mistakes within their intimate relationships. However, men with higher levels of self-compassion but lower levels of conscientiousness demonstrated less motivation to correct interpersonal mistakes. In regard to women, the authors found that conscientiousness and self-compassion were related to enhanced motivation to correct interpersonal mistakes. However, the constructs of self-compassion and conscientiousness were independent of one another (Baker \& McNulty, 2011).

In addition to these findings, Baker and McNulty (2011) found that men with both high levels of self-compassion and high levels of conscientiousness engaged in more constructive problem-solving with their spouses during observations. However, men with higher levels of self-compassion but lower levels of conscientiousness engaged in less constructive problemsolving. In regard to the women observed, self-compassion and conscientiousness were not correlated with problem-solving behavior. Men high in conscientiousness and self-compassion also were more willing to engage in accommodating behaviors, whereas men low in conscientiousness were less willing to engage in such behaviors.

Overall, Baker McNulty (2011) suggested that men endorsing higher levels of selfcompassion may require additional sources of motivation before rectifying interpersonal mistakes. However, women with higher levels of self-compassion do not appear to require additional sources of motivation. In addition, for women, self-compassion was related to 
enhanced motivation to correct problems and fewer declines in marital satisfaction. It appears that decreased self-compassion and possibly more self-criticism is an important motivator for men within interpersonal relationships. The authors suggested that self-compassion may protect women against higher stress levels stemming from their increased desires to maintain their relationships. Also, self-compassion may protect men against higher stress levels caused by the potentially high levels of achievement motivation; and men who engage in positive thoughts of self may have less motivation to correct their interpersonal mistakes (Baker \& McNulty, 2011).

Furthermore, self-compassion may be unfavorable for men if additional sources of motivators for achievement are not present. Self-compassion may benefit vocational, athletic, and academic performance in individuals with an already greater level of achievement motivation, but it may be a hindrance for people with lower levels of achievement motivation (Baker \& McNulty, 2011).

Clinical utility of self-compassion. Barnard and Curry (2011) suggested psychologists have a growing interest in developing interventions and therapeutic techniques that may increase self-compassion. Psychologists have begun developing treatments to assess whether selfcompassion can be heightened and whether or not increased levels of self-compassion enhance well-being while also minimizing dysfunction and distress. These authors noted six psychotherapeutic approaches that may enhance self-compassion, which include compassionate mind training (CMT), imagery building, gestalt two-chair technique, mindfulness-based stress reduction (MBSR), dialectal behavior therapy (DBT), and acceptance and commitment therapy (ACT).

The clinical utility of self-compassion has also been implicated in psychotherapy for individuals diagnosed with personality disorders using both short-term dynamic and cognitive 
approaches. Schanche, Stiles, McCullough, Svartberg and Nielsen (2011) found in their sample of 50 individuals diagnosed with a Cluster $\mathrm{C}$ personality disorder that increases in selfcompassion led to decreases in personality pathology, interpersonal concerns, and psychiatric symptoms. Therefore, the authors purported that self-compassion can support positive mental health in those diagnosed with Cluster $\mathrm{C}$ personality disorders.

Barnard and Curry (2011) reported that self-compassion research and self-compassion based interventions are in the early stages of development. They suggested that more research is needed to provide support for its construct validity, components, associations with well-being, and how it can be used in applied clinical practice. Therefore, it is vital to continue within this line of research to further provide evidence for the positive outcomes and correlates of selfcompassion.

\section{Current Study and Research Hypotheses}

Pepping et al. (2015) emphasized the need for continued research on the impact of parental responsiveness and the subsequent development of self-compassion in adult children. Kelly and Dupasquier (2016) noted the need for examining the impact of recalled parental warmth on later self-compassion in a more heterogeneous population beyond that of female undergraduate students.

The current study sought to add to this literature while providing further support specifically related to paternal nurturance. The aforementioned studies were a broader examination of overall parental warmth whereas this study specifically examined paternal nurturance and its relationship to levels of self-compassion. Furthermore, as lower levels of adherence to masculine norms have been found to be associated with higher levels of selfcompassion (Reilly et al., 2014), it was important to further examine how adherence to 
masculine norms and perceptions of paternal nurturance may be connected to levels of selfcompassion. This study is unique in that it specifically looked at males (e.g., the father-son dyad). It also focused on male undergraduate students - a population other than female undergraduate students as suggested by Kelly and Dupasquier (2016).

The purpose of this study was to examine whether two independent variables (i.e., conformity to masculine norms and perceptions of paternal nurturance) predicted the dependent variable (i.e., levels of self-compassion). The overall intent of the researcher was to understand if a caring relationship between father (or father figure) and son as well as the son's adherence to habits and behaviors associated with traditional masculinity in the United States predicted endorsed levels of self-compassion as reported by the participant.

The author of this study focused on these variables in order to expand the research related to masculinity, perceptions of paternal nurturance, and self-compassion. As part of this study, the following relationships among the aforementioned variables are hypothesized:

Research question 1. Does adherence to masculine norms predict levels of selfcompassion?

Hypothesis 1. Adherence to masculine norms will explain a significant amount of variance in participants' levels of self-compassion. It is expected that higher levels of adherence to masculine norms will be negatively correlated with levels of self-compassion.

Research question 2. Do perceptions of paternal nurturance predict levels of selfcompassion?

Hypothesis 2. Perceptions of paternal nurturance will explain a significant amount of variance in participants' levels of self-compassion. It is expected that higher levels of perceived paternal nurturance will be positively correlated with levels of self-compassion. 
Research question 3. Do adherence to masculine norms and perceptions of paternal nurturance predict levels of self-compassion?

Hypothesis 3. Adherence to masculine norms and perceptions of paternal nurturance will explain a significant amount of variance in participants' levels of self-compassion. It is expected that lower levels of adherence to masculine norms and perceived paternal nurturance will be significantly correlated with levels of self-compassion. 


\section{Chapter II: Methods}

\section{Research Approach}

The purpose of this study was to ascertain whether greater adherence to traditional masculine norms and levels of perceived paternal nurturance predicted levels of self-compassion in males in early adulthood at West Virginia University. Given the purpose of this study, a quantitative-descriptive design was employed and therefore it was not possible to establish a causal relationship. Inferential statistics were used in order for the researcher to draw conclusions from the acquired data.

\section{Participants}

The participants in this study were self-identified males who were currently in early adulthood. Early adulthood was defined in this study to include ages 18 to 39 years (Bigner, Grayson \& Milevsky, 2010). Participants were selected via convenience sampling from West Virginia University using the online survey system Qualtrics.

An a priori power analysis using $\mathrm{G}^{*}$ Power (Faul, Erdfelder, Buchner, \& Lang, 2009) determined that the minimum number of participants for this study was 68 when utilizing a medium effect size parameter $\left(\mathrm{f}^{2}=0.15\right)$. Eligibility criteria included the following: $(1)$ the individual identified as male, (2) the individual was within the previously mentioned age range, and (3) the individual had an established relationship with a father figure which could have included their biological father, stepfather, adoptive father, adoptive stepfather, or another individual who they considered to be their father figure. Participants provided demographic information (see Appendix D) and consent (see Appendix C) prior to completing the measures. A total of 300 participants provided responses to at least some of the items on at least one of the three measures. Of these 300 participants, 106 did not complete all of the measures required to 
be included in the final analysis. Therefore, a total of 194 participants comprised the final sample in this study.

\section{Procedures}

Prior to administering any assessments, the researcher pilot tested the administration of the three different assessments in order to gain the average duration it would take for participants to complete this study. The researcher contacted current students in the WVU Counseling Psychology Department asking them to complete a full mock administration of the three different assessments and the demographics questionnaire. The pilot subjects were also asked to report how long it took for them to complete the entire mock administration. The author attained 10 pilot administrations and took the longest duration of those 10 administrations. This time to completion (i.e., 13 minutes) served as the approximation that was included in the recruitment email for the actual study. Participants in the actual study were then informed than it should take no longer than 13 minutes to complete the study.

The study was submitted to and approved by the Institutional Review Board (IRB) at West Virginia University prior to data collection (see Appendix A). A web-based methodology was used to obtain the sample of men on a voluntary basis. The researcher posted online advertisements (please see Appendix B for advertisements) via the Daily Athenaeum (the student newspaper) website as well as the Mountaineer Information Xpress (MIX) website via the Campus Announcements section. These advertisements invited students to take part in a study about males, self-compassion, and parenting. The advertisements also included a description of enticements included in the next paragraph as well as a URL to the survey. In addition to these data collection methods, a mass email was sent out to all West Virginia University students with a MIX email account by a MIX email administrator. This mass email was sent as part of "Survey 
Tuesday" which included links to multiple studies received by WVU's University RelationsDigital. The current study was included as a link in this email in which students were voluntarily able to click on the link which would then direct them to the survey on Qualtrics. The researcher did not initiate this collection method but did inform the IRB of this. The IRB approved this collection method as well.

Enticements were utilized in this research and are described in the recruitment email. If students agreed to complete the study, they were then invited to enter their email for the chance to win one of ten $\$ 5$ Amazon e-gift cards. Participants were informed that there was a total of 10 gift cards available to win. Students who wished to participate accessed the URL provided in the advertisement that sent them to Qualtrics, the online-based survey website. After accessing the URL that led them to the Qualtrics website, participants were then prompted to read and agree to the informed consent (see Appendix C). The informed consent explained participants' rights, anonymity, confidentiality, minimal risk of discomfort during survey completion, and their ability to terminate their participation at any point during the administration. Those who wished to continue endorsed their agreement by clicking on the displayed box that confirmed their agreement to the terms and conditions explicitly stated in the informed consent. After electronically agreeing to the informed consent, participants completed a demographics questionnaire (please see Appendix D) that obtained descriptive characteristics of the participants.

Once this was completed, participants were then directed to the three separate measures used in the current study. Participants completed the Conformity to Masculine Norms Inventory46 (CMNI-46; Parent \& Moradi, 2009) consisting of 46 items and the Nurturant Fathering Scale (NFS; Finley, 1998; Williams \& Finley, 1997) consisting of nine items (please see Appendix E). 
Publishing the CMNI-46 is not permitted and therefore is not included in the appendices. They also completed the 26-item Self-Compassion Scale (SCS; Neff, 2003b) (please see Appendix F). The order in which these measures were administered was random to control for ordering effects. Participants who provided incomplete or invalid responses were excluded from the analyses.

After the participants completed the measures, they were directed to the final page which thanked them, provided debriefing, and provided them with the researcher's email address if they had any further concerns or questions. Also, this page included the option to enter the Amazon egift card drawing after completion. If a participant chose to enter the drawing, they were required to enter their email address. There was no other personal information required. Participants were notified that their email address was not linked to their survey responses. Responses were collected using Qualtrics and were only viewed by the researcher.

\section{Measures}

Conformity to Masculine Norms Inventory-46 (CMNI-46; Parent \& Moradi, 2009).

To assess conformity to masculine norms, participants completed the Conformity to Masculine Norms Inventory-46 (CMNI-46; Parent \& Moradi, 2009). The CMNI-46 is a self-report measure that contains 46 items that assess conformity to nine masculine norms. The nine masculine norms include: winning, emotional control, primacy of work, risk-taking, violence, heterosexual self-presentation, playboy, self-reliance, and power over women. Participants respond to a 4point Likert scale that ranges from $(0=$ Strongly disagree, to $3=$ Strongly agree $)$ with higher scores representing greater adherence to masculine norms.

The authors conducted a confirmatory factor analysis to evaluate whether an abridged version of the original Conformity to Masculine Norms Inventory (CMNI) (Mahalik et al., 2003) would provide adequate psychometric properties. The confirmatory factor analysis indicated that 
the CMNI-46 demonstrated adequate data-model fit on all fit indexes. However, this was after eliminating two subscales (i.e., Dominance and Pursuit of Status) because they did not meet the .60 factor loading criterion established by the authors. In addition, they found that the Cronbach's alpha coefficients remained consistent following the item reduction, ranging from .77 to .91 (Parent \& Moradi, 2009).

The CMNI-46 produced promising results in subsequent studies. Parent and Moradi (2011) examined the factor structure, reliability, and validity of the measure. The authors found acceptable fit in regard to the factor structure, $\mathrm{CFI}=.92$. Cronbach's alpha coefficients ranged from .78 to .89 , demonstrating good to excellent internal consistency reliability. Convergent validity was also assessed; findings demonstrated that the coefficients ranged from .24 to .94 when correlated with several of the subscales on the Brannon Masculinity Scale (BMS; Brannon \& Juni, 1984), the Male Role Norms Inventory (MRNI; Levant et al., 1992), and the Power over Women subscale of the Gender-Based Attitudes toward Marital Roles scale (GBATMR; Hoffman \& Kloska, 1995). Therefore, nine of the 11 subscales fell within the medium to large range according to Cohen's (1992) standards for effect sizes. In further support for this measure's internal consistency reliability, Wong et al. (2013) found the coefficient alpha to be .84 in their research utilizing the CMNI-46.

\section{Nurturant fathering}

Measuring fatherhood nurturance. Finley and Schwartz (2004) noted that the assessment of nurturant fathering has failed to keep pace with the shifts in fathering practices despite the evolving role expectations brought about by the social movements of the 1960's and 1970's. The authors suggested that there is currently and always will be a need to conceptualize and assess the behaviors, feelings, and attitudes of fathers within changing social and familial 
environments. The authors further purported that previous research has focused on the amount of time fathers spend with their children while overlooking the importance of the content and nature of the father-child relationship.

Development of the Nurturant Fathering Scale. Finley and Schwartz (2004) investigated nurturant fathering as perceived by the adolescent or adult child from a retrospective standpoint. The authors described the scale as a phenomenological measure with the ability to assess the child's lifelong perceptions of their father, which is then implicated in the child's psychosocial functioning.

In a subsequent study, Finley, Mira, and Schwartz (2008) conducted a confirmatory factor analysis that indicated a one-factor solution for nurturant fathering fit the data well, $\chi^{2}$ $(23)=169.11, p<.001 ; \mathrm{CFI}=.98 ; \mathrm{NNFI}=.97 ; \mathrm{RMSEA}=.068$. The authors reported that the standardized pattern coefficients ranged from .67 to .91 and had a mean of .77. Finley et al. (2008) replicated their findings regarding the statistical properties of the Nurturant Fathering Scale within a new sample of 1,714 young adult university students. They noted that the Cronbach's alpha coefficients were nearly identical to those yielded in the Finley and Schwartz (2004) study.

Finley et al. (2008) stated that the Nurturant Fathering Scale can be used for the exploration of family issues and various research questions that require the assessment of individuals' perceptions of their fathers. In addition, the authors noted that the scale can be used across different family forms (e.g., divorced, intact). They proposed that the scale has the potential to provide significant contributions to the family, empirical, and applied literatures.

Nurturant Fathering Scale (NFS; Finley, 1998; Williams \& Finley, 1997). In order to assess perceptions of paternal nurturance, the participants completed the NFS (Finley, 1998; 
Williams \& Finley, 1997). This self-report measure includes a total of nine items, each of which is rated on a 5-point Likert scale with higher scores representing greater perceived paternal nurturance. The items assess participants' perceptions of affective quality of paternal care provided by their father while they were growing up (Finley \& Schwartz, 2004). The participants were prompted to carefully read each item and then respond using the provided 5-point rating scale. The measure did not require any reverse scoring. Scores ranged from 9 to 45, with higher scores representing greater perceived paternal nurturance

Original studies seeking to validate this measure demonstrated Cronbach's alpha coefficients ranging from .88 to .90 (Finley, 1998; Williams \& Finley, 1997). Finley and Schwartz (2004) conducted both exploratory factor analysis (EFA) and a confirmatory factor analysis (CFA) to further extend the scale's psychometric integrity. The exploratory factor analysis found that all nine items on the scale loaded on a single factor at .76 or higher. Effect sizes ranged from .57 to .83 . Their confirmatory factor analysis showed an acceptable fit at $p<.001$. Effect sizes ranged from .50 to .85. Cronbach's alpha for Finley and Schwartz's (2004) study was .94 . The authors concluded that the NFS exhibits both reliable and internally consistent ratings of fathering in specific content domains. Finley and Schwartz (2008) found nearly identical Cronbach's alpha coefficients in a replication study focused on college-aged students, an important implication for the current study. The NFS also appears to have some evidence of cultural validity as evidenced by Doyle et al. (2011). These authors found that the NFS may be applicable to young African American individuals; however, this was after modifying the measure to a total of eight items. Doyle et al. (2015) indicated that the NFS is psychometrically appropriate for use with African American samples.

Retrospective reporting. Finley and Schwartz (2006) described the value of using 
retrospective reports in fathering research. The authors suggested that the child's retrospective perceptions of the parent strongly impact the child's current and future behavior. For example, if the adult child perceived a high level of parental involvement, then the father's influence is a result of that perception, despite the validity of the perception. In order to best attain the overall impact of father involvement, the authors purported that it is most effective to ask adults to account for their father's total involvement throughout their childhood and adolescence.

Self-compassion Scale (SCS; Neff 2003b). Self-compassion was assessed using the Self-Compassion Scale (SCS; Neff 2003b). Neff (2003b) developed the SCS in a series of pilot testing, focus groups, and studies. The SCS utilizes a 5-point Likert-type scale which ranges from 1 (almost always) to 5 (almost never). There are a total of 26 items that assess the three components of self-compassion (i.e., self-kindness, common humanity, and mindful acceptance) and their counterparts (i.e., self-judgment, isolation, and over-identification) via six subscales. Higher scores indicate greater levels of self-compassion.

Neff (2003b) utilized both an EFA and a CFA to further develop the scale and ensure appropriate psychometrics. In the exploratory factor analysis, items with loadings below .40 were eliminated from the scale. The author then conducted a confirmatory factor analysis of the 26 items remaining. The confirmatory factor analysis yielded a six-factor model which fit the data adequately well (NNFI=.90; $\mathrm{CFI}=.91)$. Each factor loading was found to be significantly different from zero $(p<.001)$. The author also conducted a single higher-order model using a CFA. The results yielded a model which fit the data marginally well (NNFI=.88; CFI=.90) (Neff, 2003b).

Furthermore, the author accounted for social desirability bias and also calculated Pearson correlation coefficients to assess construct validity that was found to be consistent with what the 
author had hypothesized. Self-compassion was found to be significantly negatively correlated with a subscale measure of self-criticism (-.65), significantly positively correlated with social connectedness (.41), and significantly positively correlated with the Attention (.11), Clarity (.43), and Repair (.55) subscales of the Trait-Meta Mood scale. In addition to these scales, Neff (2003b) noted that the SCS had a significant negative correlation with the Speilberger Trait Anxiety Inventory $(r=-.42, p<.01)$ and the Self-Rating Depression Scale $(r=-.34, p<.01)$. The SCS was found to have a significant positive correlation with the Life Satisfaction Scale $(r=.45$, $p<.01)$. Lastly, the SCS had a significant negative correlation with neurotic perfectionism that was measured by the Discrepancy subscale as part of the Almost Perfect Scale $(r=-.57, p<.01)$ (Neff, 2003b).

Furthermore, Neff (2003b) stated that the coefficient alpha of the SCS among college students was .92. In regard to construct validity, the SCS had significant positive correlations with emotional intelligence, social connectedness, and life satisfaction among college students. The SCS also had significant negative correlations with depression, anxiety, and self-criticism among college students.

\section{Data Analysis}

The collected data was examined prior to the preliminary analysis. Any missing values and/or outliers were identified. The author conducted preliminary analyses to ensure the collected data met certain assumptions (i.e., variable types, non-zero variance, multicollinearity, homoscedasticity, independent errors, normally distributed errors, independence, and linearity).

In regard to variable types, Field (2009) suggested that all predictor variables must be quantitative and the outcome variable should be quantitative, unbounded, and continuous. This study consisted of measures using Liker-type scales and thus did meet this assumption. The next assumption needed to be met was the non-zero variance. This assumption stipulates that the 
predictors should have variation in value. In order to check this assumption, the author used SPSS to produce a residuals versus fitted values plot for each of the variables. If the residuals were not scattered randomly around the zero for a given variable, then this assumption was to be considered violated.

Multicollinearity refers to the idea that there should be an imperfect relationship between the two predictor variables. Therefore, the predictor variables should not evidence a high correlation (i.e., above .80 or .90 ). In order to check this assumption, the author utilized SPSS collinearity diagnostics, specifically the variance inflation factor (VIF) and tolerance statistics. However, it should be mentioned that there are other useful measures that could have been used if this assumption was violated including variance proportions, condition indexes, uncentered cross-products matrix, and eigenvalues of the scale. If the author found that this assumption was violated, one potential solution to lessen multicollinearity would have been to collect further data and conduct the analysis again to determine the VIF. Also, if this did not lead to a solution, it may have been possible to perform a factor analysis of the predictor variables and condense them into a subset of uncorrelated factors. If the assumption was still violated, then the unreliability of the model would be acknowledged in the discussion (Field, 2009). These corrections may also have been helpful if the assumption of linearity was violated.

The assumption of homoscedasticity suggests that the variance of the residual terms should be constant for each level of the predictor variables. The author checked this assumption by visually examining the plot of standardized residuals by regression standardized predicted value using SPSS. The points should have been randomly and evenly distributed throughout the plot. If this was not the case, then this assumption would have been violated. Another option to check this assumption would have been to run the Goldfeld-Quandt, Breusch-Pagan, or Cook- 
Weisberg Tests for heteroscedasticity. If this assumption was violated there were several options. It may have been necessary to alter the specification of the model and/or transform the variables. It may have been possible to use robust standard errors, which could have altered both the standard errors and significance tests. Lastly, it may have been possible to correct for heteroscedasticity using the weighted least squares, which could have minimized a weighted sum of squared residuals (Field, 2009).

The author also checked the assumption for independent errors. Field (2009) suggested that residual terms should be uncorrelated for any two observations. The author used the DurbinWatson Test to check this assumption. Conservative estimates advise that values less than one or greater than three are problematic (Field, 2009). In addition to the Durbin-Watson Test, the author had the option to use the autocorrelation function plot or the runs test to detect residual clustering (Chatterjee \& Simonoff, 2013). If this assumption was violated, then the author planned to use several remedial techniques to transform the variables. These techniques may have included the Cochrane-Orcutt procedure (Cochrane \& Orcutt, 1949) or the Hildreth-Lu procedure (Beach \& MacKinnon, 1978).

The author also checked the assumption for normally distributed errors within the data. In accordance with this assumption, the difference between the data and the model should be zero or frequently close to zero. Also, it is important to note that the predictor variables are not required to be normally distributed. The author ensured the data were normally distributed using the Kolmogorov-Smirnov test in SPSS and observing the output of the histograms and normal PP plots which would have indicated deviation from normality. If the data violated this assumption and evidenced non-normality, it may have been necessary to transform the raw data. Another way to correct for this violation would have been to utilize robust methods (e.g., 
trimmed mean, bootstrap). More specifically, a robust regression could have been attempted if necessary (Field, 2009).

Another assumption that was checked was that of independence. According to Fields (2009), all values of the outcome variable should be independent (i.e., every value of the outcome variable must come from a separate entity). The author checked this assumption to ensure that each value of the outcome variable (i.e., level of self-compassion) came from a separate participant when examining the data for abnormalities and/or outliers. Any data violating this assumption would have been removed. There appeared to be no violations of this assumption.

Lastly, the author checked for the assumption of linearity. According to this assumption, the mean values of the outcome variable for each increment of the predictor variables should be distributed along a straight line. The author checked this assumption by again examining the residual plots of the SPSS output. More specifically, the author evaluated the histograms and normal probability plot of the residuals to ensure that the data points were evenly dispersed around zero. If there was an observed curve in these graphs, then the assumption of linearity would have been violated. If this assumption had been violated, then the author could have used a logistic regression model or discussed the limited generalizability of the predictive model within the discussion section. It is important to note that conclusions about the sample can still be inferred irrespective of the assumptions being violated, however, the findings cannot be generalized beyond the sample (Field, 2009).

In addition, descriptive statistics were calculated via SPSS to further analyze measures of central tendency and normal distribution for each variable in the data set (i.e., adherence to masculine norms, perceptions of paternal nurturance, and levels of self-compassion). The data 
were represented both graphically (e.g., histogram, scatterplots) and numerically. While these data were not used in the regression interpretation, it is a useful summary of the data. The author also used SPSS to generate a correlation matrix of the descriptives to display the correlations among the three variables. Also, the correlation matrix displays a one-tailed probability for each correlation coefficient, which can assist in identifying interrelations between the predictors (Field, 2009). This is presented in the Results chapter of this study.

It was hypothesized that perceived paternal nurturance and conformity to masculine norms would significantly predict levels of self-compassion. The data and hypothesis were analyzed and tested using a multiple linear regression. Perceptions of paternal nurturance and conformity to masculine norms served as the predictor variables whereas levels of selfcompassion served as the outcome variable. The predictor variables were entered into the model using a hierarchical (blockwise) entry. Given previous research findings evidencing a negative correlation between conformity to masculine norms and self-compassion (Reilly et al., 2014), conformity to masculine norms was entered into the model first followed by perceptions of paternal nurturance. Therefore, the present study sought to determine whether perceived paternal nurturance and levels of conformity to masculine norms predicted levels of self-compassion in a sample of college-aged men. After reviewing the literature regarding masculinity and paternal nurturance, the author selected the three assessments discussed in the measures section that were used to collect the responses from each participant. In addition, the author conducted reliability estimates on the measures using Cronbach's alpha. 


\section{Chapter III: Results}

\section{Descriptive Statistics}

The descriptive statistics of the current sample will be reported first. The original sample consisted of 300 participants. Of the original sample, 194 participants completed each item on all three of the measures required for the analysis. All 194 participants in the sample self-identified as male. Table 1 presents the ethnicity and year in school for the participants in the sample.

Table 1

Ethnicity and Year in School

\begin{tabular}{lcc}
\hline Characteristics & Number of Participants & Percentage \\
\hline Ethnicity & & \\
Non-Hispanic White or Euro-American & 166 & 85.6 \\
Black, Afro-Caribbean, or African American & 6 & 3.1 \\
Multiracial & 6 & 3.1 \\
South Asian or Indian American & 4 & 2.1 \\
Latino or Hispanic-American & 3 & 1.5 \\
East Asian or Asian American & 3 & 1.5 \\
Middle Eastern or Arab American & 2 & 1 \\
Prefer Not to Respond & 2 & 1 \\
Native American or Alaskan Native & 1 & .5 \\
Other & 1 & .5 \\
Year in School & & \\
Freshman & 16 & 8.2 \\
Sophomore & 34 & 17.5 \\
Junior & 41 & 21.1 \\
Senior & 45 & 23.2 \\
Master's Student & 26 & 13.4 \\
Doctoral Student & 19 & 9.8 \\
Professional Student (e.g., Law) & 7 & 3.6 \\
Medical Student & 6 & 3.1 \\
\hline
\end{tabular}

The average age of participants was 22.47 , ranging in years from 18 to 36 . There was a discrepancy between the required age limits reported on the informed consent and the required age limits reported on the demographics questionnaire. Participants who were not within the age range from 18 to 36 were not included in the analysis. Demographic information regarding the 
participants' parents'/caregivers' marital status, father figures' highest level of education, and fathers' relationship to participants was also collected and is displayed in Table 2 below.

Table 2

Parents'/Caregivers' Marital Status, Fathers' Education, Fathers' Relationship to Participants

\begin{tabular}{lcc}
\hline Characteristics & Number of Participants & Percentage \\
\hline Parents'/Caregivers' Martial Status & & \\
Married & 147 & 75.8 \\
Divorced/Separated & 35 & 18 \\
Widowed & 6 & 3.1 \\
Never Married and Parents Do Not Live Together & 1 & 2.1 \\
Never Married and Parents Live Together & 1 & .5 \\
Other & & .5 \\
Fathers' Education Level & 6 & 3.1 \\
Grade School & 51 & 26.3 \\
High School/GED & 12 & 6.2 \\
Vocational/Technical Degree & 9 & 4.6 \\
2-Year College Degree & 58 & 29.9 \\
4-Year College Degree & 40 & 20.6 \\
Master's Degree & 18 & 9.3 \\
Professional Degree (e.g., Law, Doctorate) & & \\
Fathers' Relationship to Participants & 179 & 92.3 \\
Biological Father & 6 & 3.1 \\
Stepfather & 1 & .5 \\
Adoptive Father & 8 & 4.1 \\
Other Father Figure (e.g., grandfather) & & \\
\hline
\end{tabular}

Participants' responses indicated that $90.2 \%$ lived in the same household as their father/father figure for the majority of their life. The average number of years the participants lived in the same household as their father/father figure was 17.88, ranging from 0 to 28 years.

Examining the descriptive statistics of the measures used in this study, the mean score of the CMNI-46 was 62.89 with a standard deviation of 15.32. Parent and Moradi (2009) found that the mean score of the CMNI-46 in their study was 66.55 with a standard deviation of 12.81 . The mean score of the NFS was 20.02 with a standard deviation of 8.14. Finley and Schwartz (2004) found that the mean score of the NFS in their study was 33.26 with a standard deviation of 9.02 
The mean score of the SCS was 76.81 with a standard deviation of 17.26. The method of calculating this mean was slightly different from how Neff (2003b) calculated the mean in her study. However, the method used to calculate the mean in the current study was per Neff's suggestion within the measure's scoring materials. Please see Table 3 for the descriptive statistics of the measures for this study.

Table 3

Descriptives of Measures

\begin{tabular}{lccccc}
\hline Measure & $\begin{array}{c}\text { Minimum } \\
\text { Statistic }\end{array}$ & $\begin{array}{c}\text { Maximum } \\
\text { Statistic }\end{array}$ & $\begin{array}{c}\text { Mean } \\
\text { Statistic }\end{array}$ & $\begin{array}{c}\text { Mean } \\
\text { Std. Error }\end{array}$ & $\begin{array}{c}\text { Std. Deviation } \\
\text { Statistic }\end{array}$ \\
\hline CMNI-46 & 25 & 106 & 62.89 & 1.1 & 15.32 \\
NFS & 9 & 45 & 20.02 & .58 & 8.14 \\
SCS & 32 & 130 & 76.81 & 1.24 & 17.26 \\
\hline
\end{tabular}

Descriptive statistics were also calculated to further analyze the distributions for each variable in this data set. Both the Kolmogorov-Smirnov and Shapiro-Wilk tests were used to perform this analysis. A Kolmogorov-Smirnov test was used to test for normality on the dependent variable of conformity to masculine norms. The CMNI-46 scores, $\mathrm{D}(194)=0.50$, $p>.05$, indicated that the data were normally distributed within this sample. The Shapiro-Wilk test, which has been shown to have more power in detecting differences from normality (Field, 2009), also demonstrated that this distribution of scores did not significantly differ from a normal distribution $\mathrm{W}(194)=.986, p>.05$.

The NFS scores, $\mathrm{D}(194)=.129, p<.05$, indicated that the data were not normally distributed within this sample. The Shapiro-Wilk test also demonstrated that this distribution of scores did significantly differ from a normal distribution $\mathrm{W}(194)=.912, p<.05$. However, Field (2009) reported that predictors in a regression analysis do not need to be normally distributed. 
Scores on the SCS, D(194) = .037, $p>.05$, were normally distributed within this sample. The Shapiro-Wilk test also demonstrated that this distribution of scores did not significantly differ from a normal distribution $\mathrm{W}(194)=.996, p>.05$.

A correlational table of the variables was also generated and is presented in Table 4, below. This table displays a one-tailed probability for each correlation coefficient. This was generated in order to identify any interrelations between the predictor variables. Table 3 demonstrates that there is not a statistically significant correlation between the two predictor variables, thus decreasing the likelihood of multicollinearity. The assumption of multicollinearity will be further discussed in the assumptions section.

Table 4

Correlations

SCS Total CMNI-46 Total NFS Total

\begin{tabular}{cccc}
\hline Pearson Correlation & 1.000 & $-.167^{*}$ & $-.178^{* *}$ \\
SCS Total & $-.167 *$ & 1.000 & -.003 \\
CMNI-46 Total & $-.178^{* *}$ & -.003 & 1.000 \\
NFS Total & & & $.007 * *$ \\
Sig. (1-tailed) &. & $.010^{*}$ & .483 \\
SCS Total & $.010^{*}$ &. &. \\
CMNI-46 Total & $.007 * *$ & .483 & \\
NFS Total & & & \\
\hline Note: $* p<.05 ; *$ * $<.01$ & & &
\end{tabular}

\section{Assumptions and Reliability of Measures}

The data meet the first assumption in that the predictor variables in this study are quantitative and the outcome variable is quantitative, unbounded, and continuous. The current study consists of measures using Likert-type scales and thus do meet this assumption. Visual examination of the residual plots demonstrates that the predictors have variation in value and 
thus meet the non-zero variance assumption. Please see Appendices G, H, and I for these residual plots.

The assumption of multicollinearity was tested using the variance inflation factor (VIF) generated by SPSS. Field (2009) suggested that the assumption of multicollinearity is violated if the VIF is above 10 or below 0.2 . The results of this analysis demonstrated a VIF of 1 , thus the assumption of multicollinearity has not been violated. In addition, after visual inspection of the plot of standardized residuals by regression standardized predicted value, the assumption of homoscedasticity was not violated.

The Durbin-Watson test was used to check the assumption of independent errors. According to the model summary within the statistical output, the Durbin-Watson statistic was 1.88, which indicates that this assumption has been met. The assumption of independence was also met given that every value of the outcome variable came from a separate participant. Lastly, linearity was verified by visual inspection of the residual plots.

In addition to checking assumptions, the author assessed levels of internal consistency for the scores on each measure within this sample. The scores on the measures used in this study demonstrated high levels of internal consistency, as determined by Cronbach's alpha. Scores on the CMNI-46 were found to be highly reliable (46 items; $\alpha=.89$ ). Scores on the Nurturant Fathering Scale demonstrated high internal consistency as well (9 items; $\alpha=.93$ ). Lastly, scores on the SCS were also found to be highly reliable (26 items; $\alpha=.91$ ). Please see Table 5 for the internal consistency reliability statistics. The norming sample Cronbach's alpha statistics in Table 5 were derived from the original instrument development studies reported in the Measurements section in this study (Finley \& Schwartz, 2004; Neff, 2003b; Parent \& Moradi, 2009). 
Table 5

Internal Consistency Reliability of Measures

\begin{tabular}{lccccc}
\hline Measure & $\begin{array}{c}\text { Number of } \\
\text { Items }\end{array}$ & $\begin{array}{c}\text { Number of } \\
\text { Participants }\end{array}$ & $\begin{array}{c}\text { Cronbach's } \\
\text { Alpha }\end{array}$ & $\begin{array}{c}\text { Internal } \\
\text { Consistency }\end{array}$ & $\begin{array}{c}\text { Norming Sample } \\
\text { Cronbach's Alpha }\end{array}$ \\
\hline CMNI-46 & 46 & 194 & .89 & Good & .88 \\
NFS & 9 & 194 & .93 & Excellent & .94 \\
SCS & 26 & 194 & .91 & Excellent & .92 \\
\hline
\end{tabular}

\section{Hypothesis Testing}

Hypothesis 1. The first hypothesis stated that adherence to masculine norms will explain a significant amount of variance in participants' levels of self-compassion. It was expected that higher levels of adherence to masculine norms would be negatively correlated with levels of selfcompassion. This hypothesis was tested using a linear regression. The predictor variable was the total score on the CMNI-46 and the criterion (dependent) variable was the total score on the SCS. The linear regression model established that scores on the CMNI-46 did statistically significantly predict participants' scores on the SCS, $F(1,194)=6.62, p<.05$. Conformity to masculine norms scores accounted for $2.8 \%$ of the explained variability in SCS scores, a small effect size according to Cohen (1988). Table 4 demonstrates that adherence to masculine norms was statistically significantly negatively correlated with levels of self-compassion.

Hypothesis 2. The second hypothesis stated that perceptions of paternal nurturance will explain a statistically significant amount of variance in participants' levels of self-compassion. It was expected that higher levels of perceived paternal nurturance would predict levels of selfcompassion. This hypothesis was also tested using a linear regression. The predictor variable was the total score on the NFS and the criterion (dependent) variable was the total score on the SCS. The linear regression model established that scores on the NFS did statistically 
significantly predict participants' scores on the SCS, $F(1,192)=6.28, p<.05$. Perceptions of paternal nurturance scores accounted for $2.7 \%$ of the explained variability in SCS scores, a small effect size according to Cohen (1988). However, Table 4 demonstrates that perceptions of paternal nurturance were statistically significantly negatively correlated with levels of selfcompassion rather than positively correlated.

Hypothesis 3. The third hypothesis stated that adherence to masculine norms and perceptions of paternal nurturance will explain a significant amount of variance in participants' levels of self-compassion. It was expected that lower levels of adherence to masculine norms and perceived paternal nurturance would significantly predict levels of self-compassion.

A hierarchical multiple regression was conducted to determine if the entry of conformity to masculine norms and then the addition of perceptions of paternal nurturance improved the prediction of self-compassion scores. The first model examined whether conformity to masculine norms could predict levels of self-compassion. The predictor variable was the total score on the CMNI-46 and the criterion (dependent) variable was the total score on the SCS. This model was statistically significant $R^{2}=.028, F(1,192)=5.53, p<.05$. The full model of conformity to masculine norms with the addition of perceptions of paternal nurturance to predict levels of selfcompassion was also statistically significant, $R^{2}=.060, F(2,191)=6.08, p<.05$; adjusted $R^{2}=$ .05 . Please see Tables 6 and 7. The predictor variables were the total score on the CMNI-46 and the total score on the NFS and the criterion (dependent) variable was the total score on the SCS. 
Table 6

Model Summary ${ }^{c}$

Model R R Square Adjusted R Square Std. Error of the Estimate Durbin-Watson

$\begin{array}{lllll}1 & .167^{\mathrm{a}} & .028 & .023 & 17.06352\end{array}$

$\begin{array}{lllll}1 & .245^{\mathrm{b}} & .060 & .050 & 16.82554\end{array}$

1.880

a. Predictors: (Constant), CMNI-46

b. Predictors: (Constant), CMNI-46, NFS

c. Dependent Variable: SCS

Table 7

ANOVA ${ }^{a}$

\begin{tabular}{lllllll}
\hline Model & Sum of Squares & df & Mean Square & F & Sig. \\
1 & Regression & 1609.911 & 1 & 1609.911 & 5.529 & $0.20^{\mathrm{b}}$ \\
& Residual & 55903.409 & 192 & 291.164 & & \\
& Total & 57513.320 & 193 & & & \\
\hline 2 & Regression & 3441.473 & 2 & 1720.736 & 6.078 & $.003^{\mathrm{c}}$ \\
& Residual & 54071.847 & 191 & 283.099 & & \\
& Total & 57513.320 & 193 & & & \\
\hline
\end{tabular}

a. Dependent Variable: SCS

b. Predictors: (Constant), CMNI-46

c. Predictors: (Constant), CMNI-46, NFS 


\section{Chapter 4}

\section{Discussion}

\section{Summary of Findings}

The purpose of this study was to determine if adherence to masculine norms (as measured by the Conformity to Masculine Norms Inventory-46 [CMNI-46]) and perceived paternal nurturance (as measured by the Nurturant Fathering Scale [NFS]) predicted levels of SelfCompassion (as measured by the Self-Compassion Scale [SCS]) in a sample of males aged 18-36 at West Virginia University. Three hypotheses were put forth to further examine the relationships among the variables.

Hypothesis 1. It was hypothesized that higher levels of adherence to masculine norms would predict lower levels of self-compassion. The analysis demonstrated that higher levels of adherence to masculine norms did statistically significantly predict lower levels of selfcompassion. It is important to note that this was a small negative effect, however (Cohen, 1988).

This finding appears to support previous research suggesting that holding oneself with self-compassion while simultaneously adhering to masculine norms can be difficult for men given that these two concepts can be incompatible (Reilly et. al., 2014). Some of the aspects of self-compassion according to Neff (2011), including being gentle with oneself, comforting oneself, and extending warm and tender feelings towards the self, may be incongruent with aspects of masculine norms measured by the CMNI-46. It may be difficult for some men to treat themselves with gentle compassion if they are attempting to live in accordance with some of the more rigid or stoic values associated with masculinity in the U.S.

To help further understand the current study's results, it is helpful to explore other theoretical perspectives related to masculinity and self-compassion. Kirby and Kirby (2017) 
suggested that masculinity and compassion (including self-compassion) could be examined using an evolutionary framework. They noted that adherence to traditional (e.g., hegemonic) masculinity puts young men at risk for negative outcomes, however, the strict adherence to masculine norms may be maintained in order to achieve status and represent one's self effectively within peer groups. The authors suggested that boys and young men adhering to these types of masculine norms (e.g., dominance, aggression, power) may be attempting to gain social rank, and thus holding others and oneself with compassion may ultimately reduce one's social status in groups of young men. It could be that the men within the current sample who evidenced higher levels of adherence to masculine norms endorsed lower levels of self-compassion because of social influence and evolutionary drives to maintain status and acceptance within the dominant male group.

This finding may also contribute to the literature showing that higher levels of adherence to masculine norms are associated with poorer mental health outcomes. Wong, Moon-Ho Ringo, Shu-Yi and Miller (2017) found in their meta-analysis that conformity to masculine norms (also measured by different versions of the CMNI) were positively associated with negative mental health and negatively related to psychological help-seeking and positive mental health. While the researchers did identify several variables in their analysis that were related to conformity to masculine norms (e.g., depression, substance use, body image concerns), they did not report on outcomes related to self-compassion. The current study adds to these findings showing that higher adherence to masculine norms may be associated with an additional factor (i.e., lower self-compassion) which can negatively relate to psychological, cognitive, and affective wellbeing (Zessin, Dickhäuser, \& Garbade, 2015). 
Hypothesis 2. It was also hypothesized that perceptions of paternal nurturance would significantly predict participants' levels of self-compassion. More specifically, it was expected that higher levels of perceived paternal nurturance would be significantly positively correlated with levels of self-compassion. It was found that perceptions of paternal nurturance did statistically significantly predict levels of self-compassion, but it was in the opposite direction of what was hypothesized. The results showed that higher levels of perceived paternal nurturance predicted lower levels of self-compassion. Again, it is important to note that this was also a small negative effect size.

This hypothesis was partially informed by previous research indicating that early experiences of warmth and nurturance from caregivers may impact individuals' ability to treat oneself with self-compassion later in life (Kelly \& Dupasquier, 2016; Pepping et. al., 2015). However, the results from the statistical analysis of this hypothesis do not appear to support this literature given that higher levels of perceived paternal nurturance actually predicted lower levels of self-compassion. These findings and unanticipated lack of support for this hypothesis may be due to several factors.

It is important to consider how the endorsement of higher levels of paternal nurturance in this study may have been impacted by the kind of nurturance the participants reported receiving from their father/father figure. Pepping et. al. (2015) suggested that the association between the type of parenting received and subsequent levels of self-compassion can be indirect and complex, lending support to the idea that there may be other factors influencing the relationship between parenting received and self-compassion. For example, in the current study, it may have been that the participants experienced paternal nurturance or involvement related more to instrumental functions (e.g., providing protection, discipline, providing financial support) rather 
than expressive functions (e.g., sharing activities, caregiving, companionship). Therefore, while higher levels of paternal nurturance may have been endorsed for some participants, their experience of the nurturance they received may have been more instrumental in nature (Finley $\&$ Schwartz, 2006). It is possible that while these participants may have experienced their father figures as nurturing, their relationship may not have entailed nurturing that would foster greater self-compassion (e.g., cultivating sense of common humanity, loving-kindness, instilling components of mindfulness) (Neff, 2003a). This finding may also be related to the expectations that sons have for their fathers in regards to fathering practices. Some of the participants in this study may have more traditional expectations of what it means to be a nurturing father (i.e., financial provider, family protector).

Therefore, when interpreting these results, it is important to consider what constitutes effective paternal nurturance that would predict higher levels of self-compassion. In their systematic review of the effects of fathering on children's developmental outcomes, Sarkadi, Kristiansson, Oberklaid, and Bremberg, (2008) suggested that fathers' active and regular engagement with their children predicted positive social, behavioral, psychological, and cognitive outcomes. However, the authors noted that what constitutes effective engagement is not well-established. A potential explanation is that the instrument used in the current study (i.e., Nurturant Fathering Scale [NFS]) may have tapped into perceived aspects of fathering that are perhaps effective, but less related to fostering self-compassion in adult children. The 9-item NFS assessed how much the participant felt their father enjoyed being a father, perceived level of father's support, perceived level of father's energy to meet the participant's needs, participant's feelings of being able to confide in their father, father's availability to spend time with the participant, emotional closeness of the father-son relationship, how well the participant got along 
with their father as a teenager, overall rating of their father, and how much of a psychological presence their father had in their daily thoughts and feelings. Therefore, while this measure may have been effective in assessing certain aspects of nurturance, it may not capture more specific expressive aspects of nurturance (Rohner \& Veneziano, 2001). When evaluating the utility and areas that the NFS assesses, Finley and Schwartz (2004) suggested, "Different fathers may express nurturance in different ways (e.g., protecting, schoolwork, sports, board games, or long walks), any or all of which children may perceive as nurturant" (p. 157).

There may also be developmental factors that could have contributed to the lack of support for this hypothesis. Neff and Pommier (2013) found that self-compassion was associated with altruism, empathic concern, and compassion for community adults but not for undergraduates within their sample. The authors hypothesized that with growth and development comes more of an understanding for suffering and the causes of suffering. Furthermore, this understanding becomes integrated within the individual and may then generalize to how they treat others and themselves. The average age in their study was 20.92 and in the current study the average age was 22.47. Given these similar mean ages, it is possible that the participants in this study may also have some difficulty with extending care to themselves, regardless of how they perceived they were treated by their fathers. Perhaps with further growth and development they will be able to further integrate their understanding of others' as well as their own suffering (Neff \& Pommier, 2013).

In addition, participants in this study were all self-identified college students. While college is a time for growth, development, and exploration, it is also a time where students face several challenges including stress, adjustment difficulties, and significant amounts of pressure from parents to succeed (Magruder \& Degges-White, 2014). Therefore, although some 
participants in this study may have perceived their fathers as nurturing, their levels of selfcompassion may have been impacted by the various demands, felt pressure to succeed from parents, and evaluative nature of college.

There may have been geographic location factors within the sample impacting this hypothesis as well. The sample was recruited from West Virginia University (WVU) whose mian campus is situated within Appalachia, approximately 75 miles from a large urban area (Theeke, Mallow, Gianni, Legg, \& Glass, 2015). It is imperative to note, however, that place of residence information was not collected on the demographic questionnaire and WVU has a diverse population of students who come from many different parts of the world. Although this cannot be stated with certainty, it is plausible that rural parenting practices and contextual factors could have partially influenced the unanticipated finding that higher levels of perceived paternal nurturance predicted lower levels of self-compassion. Parents in the Appalachian region face many potential barriers (e.g., geographic isolation, poverty, lower levels of employment) (Taubenheim \& Tiano, 2012) that may impact child-rearing practices and thus effect perceptions of paternal nurturance. However, this potential explanation should be interpreted with caution.

In addition to cultural and developmental factors, it is also important to consider the potential biological factors that may have impacted the results of this study. In their study examining the relationship between self-compassion and psychophysiological functioning, Svendsen et. al. (2016) found that higher levels of trait self-compassion significantly predicted increased ability to physiologically and psychologically regulate emotional responses. When analyzing their own results, the authors suggested that there may be several factors impacting individual differences related to self-compassion. In addition to self-compassion being impacted by early nurturing experiences, the authors also contended that self-compassion may be an innate 
trait (Svendsen et. al., 2016), though causality in this study was unknown. Therefore, if selfcompassion is an innate trait, it is plausible that there were biological factors that could have explained some variance in the current findings. Further research is needed to better understand the origins of self-compassion and the potential role of genetics.

Hypothesis 3. Overall, the results supported the main analysis, finding that levels of adherence to masculine norms and perceived paternal nurturance did predict levels of selfcompassion, with perceived paternal nurturance entered second into the hierarchical multiple regression which added a small but statistically significant contribution to the full model. Therefore, perceptions of paternal nurturance statistically significantly improved the model's ability to predict levels of self-compassion over and above levels of adherence to masculine norms alone. This finding supported the idea that a nurturing relationship between father (or father figure) and son, as well as the son's adherence to traditional masculinity in the United States, predicted levels of self-compassion within this sample.

\section{Limitations}

The results of this study should be considered preliminary given that there were several limitations. Given that the research was employed online, it was not possible to verify that all participants who took the survey fit the requirements of the study (e.g., age range, male, etc.). There may have been participants who completed the survey in order to potentially receive the incentive (i.e., Amazon eGiftcard). In addition, the self-report measure responses, specifically on the Nurturant Fathering Scale, may have been impacted by retrospective recall (Birkett, 2014) given that participants were asked to retrospectively report on perceptions of their fathers.

The results of the study may not be generalizable to the wider population given the lack of diversity within the sample (e.g., $86 \%$ identified as Non-Hispanic White or Euro-American). 
In examining self-compassion in a sample of collectivistic (Chinese) versus individualistic (American) individuals, Birkett (2014) found that Chinese students experienced the positive and negative aspects of self-compassion to a greater level than did the American students, though there were no statistically significant differences on overall SCS scores. In comparison to other research (Neff, Pisitsungkagarn, \& Hsieh, 2008), Birkett (2014) also suggested that the construct of self-compassion might differ among Eastern cultures (e.g., Taiwanese vs. Chinese) (Birkett, 2014). Therefore, given the relatively ethnic homogeneity in this sample, it may not be appropriate to generalize these findings beyond the current study. Another limitation in regard to the sample is that the majority of the participants endorsed their parental figures as having an intact marriage and therefore the findings may not be generalizable to individuals who identify having parents with other relational configurations (e.g., divorced/separated, never married and not living together, etc.).

There were also several demographic variables that were not collected which could have impacted the results. For example, sexual orientation, disability status, geographical place of residence, and religion were not collected as part of the demographic questionnaire. Homan (2014) found that participants in their study who viewed God as compassionate were more likely to engage in self-compassion themselves. She suggested that individuals who have a more secure attachment to God might be able to extend themselves kindness and approach difficulties more mindfully. Ghorbani, Watson, Kashanaki, and Chen (2017) found in their sample that Islamic seminarians more frequently identified as religious and spiritual and that those in the study who identified as both spiritual and religious were highest in self-compassion. Given that religion was not included on this study's demographic questionnaire, it is not possible to examine how this may have impacted the results. Students who identified as more spiritual or religious may have 
evidenced higher levels of self-compassion compared to other students who identified as nonreligious.

Demographic information regarding sexual orientation was also not collected, which is another limitation of this study. While the decision not to include this was based upon existing research which demonstrated that self-compassion did not vary based on sexual orientation (Lockard, Hayes, Neff, \& Locke, 2014), other research has provided some evidence that levels of self-compassion may depend on one's “outness," or level of being out as gay, lesbian, questioning, bisexual, or other. Crews and Crawford (2015) found that individuals who identified as totally out evidenced higher levels of self-compassion. Given that information about sexual orientation was not collected in the current sample, it is not possible to ascertain the impact that this variable may have had on the results. The intersectionality of these different identities (e.g., sexual orientation, religion, etc.) may have provided more context for the current findings of this study.

Another inherent limitation of this study is that the results are not generalizable to all genders. Since only males were recruited in this study, the implications do not necessarily pertain to other genders. Yarnell et al. (2015) found that men endorsed slightly higher levels of selfcompassion when compared to women. However, there have also been other studies which showed no significant differences in levels of self-compassion between men and women (Neff \& Pommier, 2013). These authors shared that their findings may indicate that gender differences in self-reported self-compassion are not consistent and may vary from sample to sample. It may have been beneficial in the current study to add to this literature and examine potential gender differences. 
There are also inherent methodological limitations associated with this study. Pepping et. al. (2015) suggested that a longitudinal design is necessary to determine whether parenting behaviors contribute to individual differences in self-compassion. The current study employed a quantitative-descriptive design using self-report measures which involved retrospective recall. Given that this was not a longitudinal study and did not implement an experimental design, it is not possible to determine a causal relationship among the variables.

Furthermore, it should be noted that the data in this study were collected from a single source (i.e., each individual participant). Marsiglio, Amato, Day, and Lamb (2000) reviewed 72 studies related to paternal involvement and child outcomes. They found that $54 \%$ of the studies collected data from a single source. They suggested that this eliminates the possibility of having other sources of objective information and these study results rely on the information provided solely by the individual participants. The current study shares this limitation in that there was no data collected from other reporters. Having other family members' (e.g., father figure's spouse, participant's sibling) retrospective reports of perceptions of paternal nurturance could have been corroborative data and may have provided further information about the level of nurturing provided. However, the importance of the participants' interpretation of their relationship to their father figures should not be understated. While other objective sources of information may have been useful, the intent of this study was to understand the subjective perceptions of the participants.

In addition to this study's design, there are also some potential limitations related to the instrumentation used in this study. There has been some recent research questioning the factor structure of the Self-Compassion Scale (Neff, 2003b). Brenner, Heath, Vogel, and Credé (2017) found that the SCS may consist of two general factors which include self-compassion and self- 
coldness. These researchers suggested that perhaps the self-coldness items should not be factored into the overall self-compassion score (Brenner et al., 2017). In a similar examination of the SCS's factor structure, Williams, Dalgleish, Karl, and Kuyken (2014) found that the measure did not meet acceptable fit criteria within a community sample. The authors concluded that the SCS is not a psychometrically robust measure of self-compassion and that further research would be necessary to develop an acceptable measure. Despite the literature supporting the psychometric properties of the SCS (Castilho, Pinto-Gouveia, \& Duarte, 2015; Neff, Whittaker, \& Karl, 2017), these findings should be taken into consideration when interpreting the results because of the potential threats to construct validity for the dependent variable in this study.

\section{Implications}

The current study has several implications despite these limitations. Given the finding that higher levels of adherence to masculine norms did statistically significantly predict lower levels of self-compassion, it is important for practitioners to help men grow in their capacity for greater self-compassion. This may be particularly important for men who ascribe to more rigid or traditional masculine norms as measured by the CMNI-46 (e.g., power over women, violence) (Parent \& Moradi, 2009). Wasylkiw and Clairo (2018) found that higher levels of selfcompassion were associated with more favorable attitudes towards help-seeking for psychological concerns. Therefore, it is important to increase men's self-compassion as this may promote greater help-seeking behavior when men are facing psychological concerns.

Therefore, it is also important for practitioners to become familiarized with specific treatments that seek to increase self-compassion. Neff and Germer (2013) developed the Mindful Self-Compassion (MSC) program which was designed to enhance self-compassion through didactics (e.g., teaching loving-kindness) as well as experiential components (e.g., formal 
mindfulness practices) in a group format. Specific treatments and programs such as MSC could be effective options for men who can benefit from increasing their levels of self-compassion.

The current study also found that higher levels of perceived paternal nurturance predicted lower levels of self-compassion. This was an unexpected finding as it was hypothesized that higher levels of perceived paternal nurturance would predict higher levels of self-compassion. While more research is certainly needed in this area, these results may have implications related to the impact of gender role conformity and parenting within the current sample. Levant, Gerdes, Alto, Jadaszewski, and McDermott (2017) suggested that fathers inform their sons' masculine gender role socialization and that there is variability in whether fathers socialize their sons to conform to traditional masculine norms more rigidly or encourage openness and flexibility. In addition, these authors noted that fathers with more rigid masculine gender role expectations for their sons may create environments that detract from healthy personal and emotional functioning and encourage adherence to traditional male roles that may lead to difficulties in adulthood.

One potential explanation for the results found in the current study is perhaps that some participants have experienced fathers who ascribed to traditional masculine norms who then instilled more traditional masculine ideals within their children. Therefore, these participants may have lower levels of self-compassion (as predicted by hypothesis 1 ). However, the participants may have still viewed fathers who conformed to traditional masculine norms as nurturing (as measured by the NFS) and perhaps this was the type of fathering expected in the current sample. Therefore, practitioners working with fathers or adult sons may want to encourage or promote more flexibility with regards to gender role conformity.

Levant et. al. (2017) purported that practitioners working with families and young children should consider exploring fathers' expectations for their sons' gender role conformity 
and masculinity. The authors noted that if fathers adhere to the belief that their role is to "toughen up and make men out of their sons" (p. 13), then practitioners should seek to potentially challenge these beliefs through the use of psychoeducation. The authors further shared the importance of psychological entities and organizations reaching out to the public and distributing information that challenges the idea of encouraging sons to adhere to rigid masculine norms (Levant et. al., 2017). The results and implications of the current study align with those of Levant et. al. (2017). It will be important for clinicians to continue encouraging more openness and flexibility in parenting practices while also celebrating the diverse and multifaceted ways in which men express their masculinity.

\section{Future Directions}

There are rich areas to build upon in order to grow the existing literature related to masculinity, fatherhood, and self-compassion. Future studies should seek to increase the multicultural diversity of their sample. It will be important to understand how culture and various identities (e.g., sexual orientation) impact the variables explored in this study. Hall, Yip, and Zárate (2016) suggested that the most effective ways of representing ethnocultural diversity in research entails using varied approaches including behavioral, biological, quantitative, and qualitative approaches. Future research should use diverse approaches when studying masculinity, fathering, and self-compassion.

It will also be important for researchers to examine psychotherapeutic factors that may lead to increased self-compassion for men who ascribe to strict traditional masculine norms. Given the findings of this study and others (Reilly et. al., 2014) showing that higher adherence to masculine norms predicted lower levels of self-compassion, it may be important for future 
researchers to identify specific therapeutic approaches or intervention strategies that foster greater self-compassion in men.

However, the first step may be to better understand how to increase men's help-seeking behavior in the first place so that self-compassion based interventions can be used with this population. In their study of 166 college-aged men, Wasylkiw and Clairo (2018) found that men who endorsed higher levels of traditional masculinity, as measured by the CMNI-46, reported that they were less likely to seek help for psychological concerns. These authors further suggested that this may be explained by men's difficulties with their tendency to self-stigmatize when considering seeking help. Since their study focused on college athletes, they supported advocacy efforts for coaches and players to become further educated on how masculine norms may impact personal development (Wasylkiw \& Clairo, 2018). Given the purpose and findings of the current study, further research should seek to better understand how campus-wide outreach or education efforts can de-stigmatize help-seeking and increase men's knowledge of how conformity to masculine norms can affect well-being.

It will be important for future researchers to examine the relationship between perceptions of paternal nurturance and levels of self-compassion given the contradictory findings between this study and previous related research (Kelly \& Dupasquier, 2016; Pepping et. al., 2015). Replication studies could help further identify if similar results are obtained in samples of men. It appears that the relationship is not clear between perceptions of paternal nurturance and levels of self-compassion and future studies should seek to use the same psychometrically sound instruments for better methodological control and to further operationalize the constructs under examination. 
Rossetto, Manning, and Green (2017) noted the importance of examining paternal support across cultures as well as over time. The authors emphasized the need to study paternal support across the lifespan over various developmental stages. In addition, Marsiglio and Roy (2012) suggested that stronger relationships between fathers and children may lead to other indirect positive outcomes (e.g., stronger relationships between resident or nonresident coparents). Therefore, it could be beneficial for future studies to examine both the direct and indirect impacts of nurturing relationships between fathers and their children, perhaps using a longitudinal or cross-sectional design.

If researchers decide to use a similar method to the one used in the current study in the future, then further validation of the Nurturant Fathering Scale may be necessary. While there have been previous studies evidencing the validity and reliability of this measure (Doyle et al. 2011; Finley, 1998; Finley \& Schwartz, 2004; Finley \& Schwartz, 2008; Williams \& Finley, 1997), there appears to be somewhat limited studies using this specific measure. Therefore, it will be important for future researchers to continue providing psychometric support for this instrument.

Future research should also approach the study of masculinity using more strengths-based methods. Rather than focusing on the negative aspects of masculinity or what men may be lacking, researchers should seek to identify those factors that are positive and contribute to men's overall well-being (i.e., self-compassion). One potential framework is the positive psychology/positive masculinity (PPPM) model (Kiselica \& Englar-Carlson, 2010). The model underscores the constructive aspects of masculinity including generative fatherhood, relational styles, male ways of caring, male courage, the group orientation of boys and men, etc. Future research can seek to understand the relationship between conformity to masculine norms and the 
various positive aspects of masculinity, including generative fatherhood and paternal nurturance. Furthermore, future research can also examine how self-compassion may actually be a strength of men.

\section{Conclusion}

The findings of the present study support previous research showing that higher levels of adherence to masculine norms may predict lower levels of self-compassion in men. Surprisingly, it was also found that higher levels of perceived paternal nurturance predicted lower levels of self-compassion. Overall, the hierarchical regression analysis showed that levels of adherence to masculine norms and perceived paternal nurturance predicted levels of self-compassion. Perceptions of paternal nurturance statistically significantly improved the model's ability to predict levels of self-compassion over and above that predicted by levels of adherence to masculine norms alone, though in the opposite direction of what was initially hypothesized. In sum, findings supported the idea that retrospective self-reports of a nurturing relationship between father (or father figure) and son as well as the son's self-reported adherence to traditional masculine norms in the United States predicted levels of self-reported selfcompassion within this sample. As previously reviewed, these findings have several theoretical as well as clinical implications for working with men. Future research should continue to obtain diverse samples of men to further substantiate these and related findings. 


\section{References}

Addis, M. E., Mansfield, A. K., \& Syzdek, M. R. (2010). Is “masculinity” a problem?: Framing the effects of gendered social learning in men. Psychology of Men \& Masculinity, 11(2), 77-90. doi:10.1037/a0018602

Addis, M. E., \& Mahalik, J. R. (2003). Men, masculinity, and the contexts of help seeking. American Psychologist, 58(1), 5.

Arrindell, W. A., Sanavio, E., Aguilar, G., Sica, C., Hatzichristou, C., Eisemann, M., Recinos, L. A., Gaszner, P., Peter, M., Battagliese, G., Kállai, J., van der Ende, J. (1999). The development of a short form of the EMBU: Its appraisal with students in Greece, Guatemala, Hungary and Italy. Personality and Individual Differences, 27, 613-628.

Baker, L. R., \& McNulty, J. K. (2011). Self-compassion and relationship maintenance: The moderating roles of conscientiousness and gender. Journal of Personality and Social Psychology, 100(5), 853-873. doi:10.1037/a0021884

Barnard, L., \& Curry, J. (2011). Self-compassion: Conceptualizations, correlates, \& interventions. Review of General Psychology, 15(4), 289-303. doi:10.1348/147608306X146086

Beach, C., \& MacKinnon, J. (1978). A maximum likelihood procedure for regression with autocorrelated errors. Econometrica, 46(1), 51-58. Retrieved from http://www.jstor.org/stable/1913644 doi:1

Berger, J. L., Addis, M. E., Green, J. D., Mackowiak, C., \& Goldberg, V. (2013). Men’s reactions to mental health labels, forms of help-seeking, and sources of help-seeking advice. Psychology of Men \& Masculinity, 14(4), 433-443. doi:10.1037/a0030175 
Bigner, J. J., Grayson, T., Milevsky, A. (2010). Understanding foundations in human development. Redding, CA: BVT Publishing.

Birkett, M. A. (2014). Self-compassion and empathy across cultures: Comparison of young adults in China and the United States. International Journal of Research Studies in Psychology, 3, 25-34. doi:10.5861/ijrsp.2013.551

Bonney, J. F., Kelley, M. L., \& Levant, R. F. (1999). A model of fathers' behavioral involvement in child care in dual-earner families. Journal of Family Psychology, 13(3), 401-415.

Boysen, G., Ebersole, A., Casner, R., \& Coston, N. (2014). Gendered mental disorders:

Masculine and feminine stereotypes about mental disorders and their relation to stigma. Journal of Social Psychology, 154(6), 546-565. doi:10.1080/00224545.2014.953028

Brannon, R., \& Juni, S. (1984). A scale for measuring attitudes about masculinity. Psychological Documents, 14, 6-7 (ms2612).

Brenner, R. E., Heath, P. J., Vogel, D. L., \& Credé, M. (2017). Two is more valid than one: Examining the factor structure of the Self-Compassion Scale (SCS). Journal Of Counseling Psychology, 64(6), 696-707. doi:10.1037/cou0000211

Castilho, P., Pinto-Gouveia, J., \& Duarte, J. (2015). Evaluating the multifactor structure of the long and short versions of the Self-Compassion Scale in a clinical sample. Journal Of Clinical Psychology, 71(9), 856-870. doi:10.1002/jclp.22187

Chatterjee, S., \& Simonoff, J. S. (2013). Wiley handbooks in applied statistics: Handbook of regression analysis (1). Somerset, NJ: Wiley.

Chu, J. Y. (2014). Supporting boys' healthy resistance to masculine norms. Psychology of Men and Masculinity, 15(3), 253-255. doi:10.1037/a0037275 
Cochrane, D., \& Orcutt, G. H. (1949). Application of least squares regression to relationships containing auto-correlated error terms. Journal of the American Statistical Association, 44, 245, 32-61.

Cohen, J. (1988). Statistical power analysis for the behavioral sciences (2nd ed.). Hillsdale, NJ: Erlbaum.

Cohen, J. (1992). A power primer. Psychological Bulletin, 112, 155- 159.

Courtenay, W. H. (2000). Constructions of masculinity and their influence on men's well-being: A theory of gender and health. Social Science \& Medicine, 50(10), 1385.

Crews, D., \& Crawford, M. (2015). Exploring the role of being out on a queer person's selfcompassion. Journal Of Gay \& Lesbian Social Services, 27(2), 172186. doi:10.1080/10538720.2015.1022272

Dearden, K., Crookston, B., Madanat, H., West, J., Penny, M., \& Cueto, S. (2013). What difference can fathers make? Early paternal absence compromises Peruvian children's growth. Maternal \& Child Nutrition, 9(1), 143-154. doi:10.1111/j.17408709.2011.00347.x

Doyle, O., Pecukonis, E., \& Harrington, D. (2011). The Nurturant Fathering Scale: A confirmatory factor analysis with an African American sample of college students. Research on Social Work Practice, 21(3), 319-327. doi:10.1177/1049731510377635

Doyle, O., Pecukonis, E. V., \& Lindsey, M. A. (2015). Correlates and consequences of father nurturance in an African American college sample. Journal of Family Issues, 36(7), 880901. doi:10.1177/0192513X13501665 
Echabe, A. E. (2010). Role identities versus social identities: Masculinity, femininity, instrumentality and communality. Asian Journal of Social Psychology, 13(1), 30-43. doi:10.1111/j.1467-839X.2010.01298.x

Faul, F., Erdfelder, E., Buchner, A., \& Lang, A.G. (2009). Statistical power analyses using G*Power 3.1: Tests for correlation and regression analyses. Behavior Research Methods, 41, 1149-1160. doi:10.3738/BRM.41.4.1149

Finley, G. E. (1998). Parental age and parenting quality as perceived by late adolescents. Journal of Genetic Psychology, 159, 505-506.

Finley, G. E., Mira, S. D., \& Schwartz, S. J. (2008). Perceived paternal and maternal involvement: Factor structures, mean differences, and parental roles. Fathering: A Journal of Theory, Research, and Practice about Men as Fathers, 6(1), 62-82. doi:10.3149/fth.0601.62

Finley, G. E., \& Schwartz, S. J. (2004). The Father Involvement and Nurturant Fathering Scales: Retrospective measures for adolescent and adult children. Educational and Psychological Measurement, 64(1), 143-164. doi:10.1177/0013164403258453

Finley, G. E., \& Schwartz, S. J. (2006). Parsons and Bales revisited: Young adult children's characterization of the fathering role. Psychology of Men \& Masculinity, 7(1), 42-55. doi:10.1037/1524-9220.7.1.42

Finley, G. E., \& Schwartz, S. J. (2008). Perceived paternal and maternal involvement: Factor structures, mean differences, and parental roles. Fathering: A Journal of Theory, Research, \& Practice about Men as Fathers, 6(1), 62-82. doi:10.3149/fth.0601.62 
Flouri, E. (2004). Correlates of parents' involvement with their adolescent children in restructured and biological two-parent families: The role of child characteristics. International Journal Of Behavioral Development, 28(2), 148-156.

Galovan, A. M., Holmes, E. K., Schramm, D. G., \& Lee, T. R. (2014). Father involvement, father-child relationship quality, and satisfaction with family work: Actor and partner influences on marital quality. Journal of Family Issues, 35(13), 1846-1867. doi:10.1177/0192513X13479948

Ghorbani, N., Watson, P. J., Kashanaki, H., \& Chen, Z. J. (2017). Diversity and complexity of religion and spirituality in Iran: Relationships with self-compassion and selfforgiveness. International Journal For The Psychology Of Religion, 27(4), 157-171. doi:10.1080/10508619.2017.1340100

Glass, J., \& Owen, J. (2010). Latino fathers: The relationship among machismo, acculturation, ethnic identity, and paternal involvement. Psychology of Men \& Masculinity, 11(4), 251261. doi:10.1037/a0021477

Gordon, D. M., Hawes, S. W., Reid, A. E., Callands, T. A., Magriples, U., Divney, A., Kershaw, T. (2013). The many faces of manhood: Examining masculine norms and health behaviors of young fathers across race. American Journal of Men's Health, 7(5), 394401. http://doi.org/10.1177/1557988313476540

Hall, G. N., Yip, T., \& Zárate, M. A. (2016). Disciplinary perspectives on multicultural research: Reply to Dvorakova (2016) and Yakushko et al. (2016). American Psychologist, 71(9), 892-893. doi:10.1037/amp0000124 
Hansen, J. A., Weissbrod, C., Schwartz, D. D., \& Taylor, W. P. (2012). Paternal involvement in pediatric Type 1 diabetes: Fathers' and mothers' psychological functioning and disease management. Families, Systems, \& Health, 30(1), 47-59. doi:10.1037/a0027519

Heath, P. J., Brenner, R. E., Vogel, D. L., Lannin, D. G., \& Strass, H. A. (2017). Masculinity and barriers to seeking counseling: The buffering role of self-compassion. Journal of Counseling Psychology, 64(1), 94-103. doi:10.1037/cou0000185

Hoffman, L. W., \& Kloska, D. D. (1995). Parents' gender-based attitudes toward marital roles and child-rearing: Development and validation of new measures. Sex Roles, 32, 273-295. doi:10.1007/BF01544598

Homan, K. J. (2014). A mediation model linking attachment to God, self-compassion, and mental health. Mental Health, Religion \& Culture, 17(10), 977-

989. doi:10.1080/13674676.2014.984163

Irons, C., Gilbert, P., Baldwin, M. W., Baccus, J. R., \& Palmer, M. (2006). Parental recall, attachment relating and self-attacking/self-reassurance: Their relationship with depression. British Journal of Clinical Psychology, 45(3), 297-308. doi:10.1348/014466505X68230

Kelly, A. C., \& Dupasquier, J. (2016). Social safeness mediates the relationship between recalled parental warmth and the capacity for self-compassion and receiving compassion. Personality \& Individual Differences, 89, 157-161. doi:10.1016/j.paid.2015.10.017

Kirby, J. N., \& Kirby, P. G. (2017). An evolutionary model to conceptualise masculinity and compassion in male teenagers: A unifying framework. Clinical Psychologist, 21(2), 7489. doi:10.1111/cp.12129 
Kiselica, M. S., \& Englar-Carlson, M. (2010). Identifying, affirming, and building upon male strengths: The positive psychology/positive masculinity model of psychotherapy with boys and men. Psychotherapy: Theory, Research, Practice, Training, 47(3), 276-287. doi:10.1037/a0021159

Leary, M. R., Tate, E. B., Adams, C. E., Batts Allen, A., \& Hancock, J. (2007). Self-compassion and reactions to unpleasant self-relevant events: The implications of treating oneself kindly. Journal of Personality and Social Psychology, 92(5), 887-904.

Levant, R. F. (2011). Research in the psychology of men and masculinity using the gender role strain paradigm as a framework. American Psychologist, 66(8), 765-776.

Levant, R. F., Gerdes, Z. T., Alto, K. M., Jadaszewski, S. E., \& McDermott, R. C. (2017). Development and evaluation of the fathers' expectations about sons' masculinity scale (short form). Psychology Of Men \& Masculinity, doi:10.1037/men0000108

Levant, R. F., Hirsh, L., Celantano, E., Cozza, T., Hill, S., MacEcheron, M., \& Schnedeker, J. (1992). The male role: An investigation of contemporary norms. Journal of Mental Health Counseling, 14, 325-337.

Levant, R. F., \& Richmond, K. (2007). A review of research on masculinity ideologies using the Male Role Norms Inventory. Journal of Men's Studies, 15(2), 130-146.

Lewis, C., \& Lamb, M. E. (2003). Fathers' influences on children's development: The evidence from two-parent families. European Journal of Psychology of Education - EJPE (Instituto Superior De Psicologia Aplicada), 18(2), 212-228.

Liu, W. M. (2005). The study of men and masculinity as an important multicultural competency consideration. Journal Of Clinical Psychology, 61(6), 685-697. doi:10.1002/jclp.20103 
Liu, W. M. (2016). Masculinities on the verge: An editorial. Psychology Of Men \& Masculinity, 17(1), 1-2. doi:10.1037/men0000020

Lockard, A. J., Hayes, J. A., Neff, K., \& Locke, B. D. (2014). Self-compassion among college counseling center clients: An examination of clinical norms and group differences. Journal of College Counseling, 17(3), 249-259. doi:10.1002/j.2161-1882.2014.00061.x

Maccoby, E. E. (1992). The role of parents in the socialization of children: An historical overview. Developmental Psychology, 28(6), 1006-1017. doi: 10.1037/00121649.28.6.1006

Magruder, J., \& Degges-White, S. (2014). Counseling concerns over the college academic year. In S. Degges-White \& C. Borzumato-Gainey (Eds.), College student mental health counseling: A developmental approach (pp. 13-28). New York, NY: Springer Publishing Company, LLC.

Mahalik, J. R., Locke, B. D., Ludlow, L. H., Diemer, M. A., Scott, R. P., Gottfried, M., et al. (2003). Development of the Conformity to Masculine Norms Inventory. Psychology of Men \& Masculinity, 4, 3- 25. doi: 10.1037/1524-9220.4.1.3

Mankowski, E. S., \& Maton, K. I. (2010). A community psychology of men and masculinity: Historical and conceptual review. American Journal of Community Psychology, 45(1/2), 73-86. doi:10.1007/s10464-009-9288-y

Marsiglio, W., Amato, P., Day, R. D., \& Lamb, M. E. (2000). Scholarship on fatherhood in the 1990s and beyond. Journal Of Marriage \& Family, 62(4), 1173-1191.

Marsiglio, W., \& Roy, K. (2012). Nurturing Dads: Fatherhood initiatives beyond the wallet. New York, NY: Russell Sage Foundation. 
Neff, K. D. (2003a). Self-compassion: An alternative conceptualization of a healthy attitude toward oneself. Self \& Identity, 2(2), 85.

Neff, K. D. (2003b). Development and validation of a scale to measure self-compassion. Self \& Identity, 2, 223-250.

Neff, K. D. (2011). Self-compassion: The proven power of being kind to yourself. New York, NY: HarperCollins.

Neff, K. D., \& Germer, C. K. (2013). A pilot study and randomized controlled trial of the mindful self-compassion program. Journal Of Clinical Psychology, 69(1), 28-44. doi: $10.1002 /$ jclp.21923

Neff, K. D., \& McGehee, P. (2010). Self-compassion and psychological resilience among adolescents and young adults. Self \& Identity, 9(3), 225-240. doi:10.1080/15298860902979307

Neff, K. D., Pisitsungkagarn, K., \& Hsieh, Y. P. (2008). Self-compassion and self-construal in the United States, Thailand, and Taiwan. Journal of Cross-Cultural Psychology, 39(3), 267-285. doi.org/10.1177/0022022108314544

Neff, K. D., \& Pommier, E. (2013). The Relationship between self-compassion and otherfocused concern among college undergraduates, community adults, and practicing meditators. Self \& Identity, 12(2), 160-176. doi:10.1080/15298868.2011.649546

Neff, K. D., Whittaker, T.A., \& Karl, A. (2017). Examining the factor structure of the SelfCompassion Scale in four distinct populations: Is the use of a total scale score justified? Journal of Personality Assessment, 99(6), 596-607. doi:10.1080/00223891.2016.1269334 
Nguyen, C. M., Ming Liu, W., Hernandez, J. O., \& Stinson, R. (2012). Problem-solving appraisal, gender role conflict, help-seeking behavior, and psychological distress among men who are homeless. Psychology of Men \& Masculinity, 13(3), 270-282. doi:10.1037/a0025523

O’Neil, J. M. (2012). The psychology of men. In E. M. Altmaier \& J. C. Hansen (Eds.), The Oxford handbook of counseling psychology (pp. 375-408). New York, NY: Oxford University Press, Inc.

O'Neil, J. M. (2013). Gender role conflict research 30 years later: An evidence-based diagnostic schema to assess boys and men in counseling. Journal of Counseling \& Development, 91(4), 490-498. doi:10.1002/j.1556-6676.2013.00122.x

Palkovitz, R. (2002). Involved fathering and men's adult development: Provisional balances. Mahwah, NJ: Lawrence Erlbaum Associates, Inc., Publishers

Parent, M. C., \& Moradi, B. (2009). Confirmatory factor analysis of the Conformity to Masculine Norms Inventory and development of the Conformity to Masculine Norms Inventory-46. Psychology of Men \& Masculinity, 10(3), 175-189. doi:10.1037/a0015481

Parent, M. C., \& Moradi, B. (2011). An abbreviated tool for assessing conformity to masculine norms: Psychometric properties of the Conformity to Masculine Norms Inventory-46. Psychology of Men \& Masculinity, 12(4), 339-353. doi:10.1037/a0021904

Parent, M. C., Moradi, B., Rummell, C. M., \& Tokar, D. M. (2011). Evidence of construct distinctiveness for conformity to masculine norms. Psychology of Men \& Masculinity, 12(4), 354-367. doi:10.1037/a0023837 
Patsiopoulos, A. T., \& Buchanan, M. J. (2011). The practice of self-compassion in counseling: A narrative inquiry. Professional Psychology: Research and Practice, 42(4), 301-307. doi:10.1037/a0024482

Pepping, C. A., Davis, P. J., O'Donovan, A., \& Pal, J. (2015). Individual differences in selfcompassion: The role of attachment and experiences of parenting in childhood. Self and Identity, 14(1), 104-117. doi:10.1080/15298868.2014.955050

Potter, R. F., Keong, Y., Francis, A. P., \& Schuster, S. (2014). Self-Compassion mediates the relationship between parental criticism and social anxiety. International Journal of Psychology \& Psychological Therapy, 14(1), 33-43.

Reidy, D. E., Shirk, S. D., Sloan, C. A., \& Zeichner, A. (2009). Men who aggress against women: Effects of feminine gender role violation on physical aggression in hypermasculine men. Psychology of Men \& Masculinity, 10(1), 1-12. doi: $10.1037 / \mathrm{a} 0014794$

Reilly, E. D., Rochlen, A. B., \& Awad, G. H. (2014). Men's self-compassion and self-esteem: The moderating roles of shame and masculine norm adherence. Psychology of Men \& Masculinity, 15(1), 22-28. doi:10.1037/a0031028

Rohner, R. P., \& Veneziano, R. A. (2001). The importance of father love: History and contemporary evidence. Review of General Psychology, 5(4), 382-405. doi:10.1037/1089-2680.5.4.382

Rossetto, K. R., Manning, J., \& Green, E. W. (2017). Perceptions of paternal support after transitioning to college: Interpretations based on the generative fathering framework. Western Journal Of Communication, 81(4), 405-425. doi:10.1080/10570314.2017.1283047 
Rummell, C. M., \& Levant, R. F. (2014). Masculine gender role discrepancy strain and selfesteem. Psychology of Men \& Masculinity, 15(4), 419-426. doi:10.1037/a0035304

Sarkadi, A., Kristiansson, R., Oberklaid, F., \& Bremberg, S. (2008). Fathers' involvement and children's developmental outcomes: A systematic review of longitudinal studies. Acta Paediatrica, 97(2), 153-158. doi:10.1111/j.1651-2227.2007.00572.x

Schwartz, S., \& Finley, G. (2005). Fathering in intact and divorced families: Ethnic differences in retrospective reports. Journal of Marriage and Family, 67(1), 207-215. Retrieved from http://www.jstor.org/stable/3600146

Schwartz, S. J., \& Finley, G. E. (2006). Father involvement, nurturant fathering, and young adult psychosocial functioning: Differences among adoptive, adoptive stepfather, and nonadoptive stepfamilies. Journal of Family Issues, 27(5), 712-731.

Schwartz, S. J., Zamboanga, B. L., Ravert, R. D., Kim, S. Y., Weisskirch, R. S., Williams, M. K., \& Finley, G. E. (2009). Perceived parental relationships and health-risk behaviors in college-attending emerging adults. Journal of Marriage \& Family, 71(3), 727-740. doi:10.1111/j.1741-3737.2009.00629.x

Schanche, E., Stiles, T. C., McCullough, L., Svartberg, M., \& Nielsen, G. H. (2011). The relationship between activating affects, inhibitory affects, and self-compassion in patients with Cluster C personality disorders. Psychotherapy, 48(3), 293-303. doi:10.1037/a0022012

Sierra Hernandez, C. A., Han, C., Oliffe, J. L., \& Ogrodniczuk, J. S. (2014). Understanding helpseeking among depressed men. Psychology of Men \& Masculinity, 15(3), 346-354. doi:10.1037/a0034052 
Smeets, E., Neff, K., Alberts, H., \& Peters, M. (2014). Meeting suffering with kindness: Effects of a brief self-compassion intervention for female college students. Journal of Clinical Psychology, 70(9), 794-807. doi:10.1002/jclp.22076

Svendsen, J. L., Osnes, B., Binder, P.-E., Dundas, I., Visted, E., Nordby, H., Sørensen, L. (2016). Trait self-compassion reflects emotional flexibility through an association with high vagally mediated heart rate variability. Mindfulness, 7(5), 1103-1113. http://doi.org/10.1007/s12671-016-0549-1

Taubenheim, A., \& Tiano, J. D. (2012). Rationale and modifications for implementing parentchild interaction therapy with rural Appalachian parents. Journal Of Rural Mental Health, 36(2), 16-26. doi:10.1037/h0095811

Theeke, L. A., Mallow, J., Gianni, C., Legg, K., \& Glass, C. (2015). The experience of older women living with loneliness and chronic conditions in Appalachia. Journal Of Rural Mental Health, 39(2), 61-72. doi:10.1037/rmh0000029

Thompson, E. H., and J. H. Pleck. 1995. “'Masculinity Ideologies: A Review of Research Instrumentation on Men and Masculinities.' In A New Psychology of Men, edited by R. F. Levant and W. S. Pollack, 129-63. New York, NY: Basic Books.

U.S. Census Bureau. (2014). Father's Day [press release]. Retrieved from https://www.census.go v/content/dam/Census/newsroom/facts-for-features/2014/cb14-ff14_fathersday.pdf

Vogel, D. L., Heimerdinger-Edwards, S. R., Hammer, J. H., \& Hubbard, A. (2011). “Boys don't cry": Examination of the links between endorsement of masculine norms, self-stigma, and help-seeking attitudes for men from diverse backgrounds. Journal of Counseling Psychology, 58(3), 368-382. doi:10.1037/a0023688 
Wasylkiw, L., \& Clairo, J. (2018). Help seeking in men: When masculinity and self-compassion collide. Psychology of Men \& Masculinity, 19(2), 234-242. doi:10.1037/men0000086

Wei, M., Liao, K. Y., Ku, T., \& Shaffer, P. A. (2011). Attachment, self-compassion, empathy, and subjective well-being among college students and community adults. Journal of Personality, 79(1), 191-221. doi:10.1111/j.1467-6494.2010.00677.x

West, C., \& Zimmerman, D. (1987). Doing gender. Gender and Society, 1(2), 125 - 151

Williams, M. J., Dalgleish, T., Karl, A., \& Kuyken, W. (2014). Examining the factor structures of the Five Facet Mindfulness Questionnaire and the Self-Compassion Scale. Psychological Assessment, 26(2), 407-418. doi:10.1037/a0035566

Williams, S. M., \& Finley, G. E. (1997). Father contact and perceived affective quality of fathering in Trinidad. Interamerican Journal of Psychology, 31, 315-319.

Wong, Y. J., Hickman, S. J., Lafollette, J. R., Shea, M., Cruz, N., \& Boghokian, T. (2013). The Subjective Masculinity Stress Scale: Scale development and psychometric properties. Psychology of Men \& Masculinity, 14(2), 148-155. doi:10.1037/a0027521

Wong, C. Y., \& Mak, W. S. (2013). Differentiating the role of three self-compassion components in buffering cognitive-personality vulnerability to depression among Chinese in Hong Kong. Journal of Counseling Psychology, 60(1), 162-169. doi:10.1037/a0030451

Wong, Y. J., Moon-Ho Ringo, H., Shu-Yi, W., \& Miller, I. K. (2017). Meta-analyses of the relationship between conformity to masculine norms and mental health-related outcomes. Journal of Counseling Psychology, 64(1), 80-93. doi:10.1037/cou0000176

Yarnell, L. M., Stafford, R. E., Neff, K. D., Reilly, E. D., Knox, M. C., \& Mullarkey, M. (2015). Meta-analysis of gender differences in self-compassion. Self \& Identity, 14(5), 499-520. doi:10.1080/15298868.2015.1029966 
Yousaf, O., Popat, A., \& Hunter, M. S. (2015). An investigation of masculinity attitudes, gender, and attitudes toward psychological help-seeking. Psychology of Men \& Masculinity, 16 (2), 234-237. doi:10.1037/a0036241.

Zessin, U., Dickhäuser, O., \& Garbade, S. (2015). The relationship between self-compassion and well-being: A meta-analysis. Applied Psychology: Health \& Well-Being, 7(3), 340364. doi:10.1111/aphw.12051

Zhang, B., Zhao, F., Ju, C., \& Ma, Y. (2015). Paternal involvement as protective resource of adolescents' resilience: Roles of male gender-role stereotype and gender. Journal of Child \& Family Studies, 24(7), 1955-1965. doi:10.1007/s10826-014-9995-3 


\section{Appendix A}

\section{IRB Approval}

IRB protocol number: 1704542939

Title: Adherence to Masculine Norms and Perceived Paternal Nurturance as Predictors of SelfCompassion

PI: Jeffrey A Daniels

The West Virginia University Institutional Review Board approved the above-referenced protocol on 22-May-2017. To access this protocol, click on the protocol number link provided. Your approval correspondence concerning this action can be found in the correspondence section HERE. Any future protocol action requests can be completed through the WVU+kc system. NEED HELP? The Office of Research Integrity and Compliance is here to assist you from initial submission of a protocol through approval and all subsequent actions. If you have any questions, please contact the Office of Research Integrity and Compliance at 304-293-7073 or email IRB@mail.wvu.edu. Thank you. 


\section{Appendix B}

\section{Online Advertisement on the Daily Anthenaeum website at: http://www.thedaonline.com/}

"Seeking male WVU students between the ages of 18-39 to complete online survey research for a chance to win one of ten \$5 Amazon eGift Cards. The study is being conducted by Dr. Jeffrey

Daniels and Steven Craig within the Department of Counseling, Rehabilitation Counseling, and Counseling Psychology. WVU's acknowledgement of this ad is on file. Please click on the following link to participate: http://wvu.qualtrics.com/jfe/form/SV_ODIKA4HwNg7fWmh"

Mountaineer Information Xpress (MIX) Campus Announcements Advertisement

\section{[Date of the post]}

If you are male WVU student between the ages of 18-39 and have a person you consider to be a father figure you could have a chance to win one of ten $\$ 5$ Amazon eGift Cards by completing online survey research about masculinity, self-compassion, and relationships. The study is being conducted by Dr. Jeffrey Daniels and Steven Craig within the Department of Counseling, Rehabilitation Counseling, and Counseling Psychology. WVU's acknowledgement of this ad is on file. Please click on the following link to participate:

http://wvu.qualtrics.com/jfe/form/SV_ODIKA4HwNg7fWmh 


\section{Appendix C}

\section{Informed Consent}

Please consider the following prior to participating in this research study: You are invited to participate in the following study about males, self-compassion, and parenting for a chance to win one of ten $\$ 5$ Amazon eGift Cards. Completing the survey should take no longer than approximately 13 minutes. Please be aware that you must be a male WVU student between the ages of 18-39 to participate. This research study was approved by the West Virginia University Institutional Review Board and is part of Steven Craig's doctoral dissertation at WVU. This research study is being advised by Dr. Jeffrey Daniels of the Department of Counseling, Rehabilitation Counseling and Counseling Psychology. Please note that your participation in this study is voluntary. While there are no anticipatory risks involved beyond the possibility of some questions raising discomfort, you may withdraw from taking the survey at any point in time. Your responses to these questions will be kept confidential. The information collected from you will be kept secure. If you have any questions or concerns, please send an email to Steven Craig (sjcraig@mix.wvu.edu) and/or Dr. Jeffrey Daniels (Jeffrey.Daniels@mail.wvu.edu). Please click confirm your participation status below if you have read and understand these explanations that have been provided to you. Thank you for your participation.

Please confirm your voluntary participation below.

I agree to participate in this study and confirm that I am a male WVU student between the ages of 18-39. 


\section{Apendix D}

\section{Demographic Questionnaire}

Please enter you age in years below (Must be between the ages 18-36 to participate)

What is your gender? (Must identify as male to participate)

O Male

O Female

Non-binary/third gender

Please indicate your race/ethnicity (please choose all that apply)

$\square$ Non-Hispanic White or Euro-American

- Black, Afro-Caribbean, or African American

Latino or Hispanic American

$\square$ East Asian or Asian American

$\square$ South Asian or Indian American

$\square$ Middle Eastern or Arab American

$\square$ Native American or Alaskan Native

$\square$ Multiracial

$\square$ Other (please indicate below)

$\square$ Prefer not to respond

If you marked other, please indicate your race/ethnicity below

Please indicate your father's relationship to you (or who you consider to be your closest male parental figure).

O Biological father

Stepfather

Adoptive father

Other father figure (e.g., grandfather)

If other, please indicate your father's or father figure's status below:

Have you lived in the same household as your father or father figure for the majority of your life?

O Yes

No

Please indicate the number of years you have lived with your father or father figure below. 
What is your classification in college?

O Freshman

Sophomore

O Junior

O Senior

O Master's Student

O Doctoral Student

Professional Student (e.g., Law)

Medical Student

Please indicate the highest level of education your father has completed.

O Grade school

O High School/GED

V Vocational/Technical Degree

2-year college degree

4-year college degree

O Masters degree

Professional degree (e.g., J.D.,M.D., Ph.D.)

Please indicate your parent(s)'/caregiver(s)' marital status.

O Married

Divorced/Separated

O Never Married and Both Parents Living Together

O Never Married and Parents Are Not Living Together

O Widowed

Other

If other, please indicate your parent(s)'/caregiver(s)' marital status below: 


\section{Appendix E}

Nurturant Fathering Scale

1. How much do you think your father enjoyed being a father?

A great deal

Very much

Somewhat

A little

Not at all

2. When you needed your father's support, was he there for you?

Always there for me

Often there for me

Sometimes there for me

Rarely there for me

Never there for me

3. Did your father have enough energy to meet your needs?

Always
Often
Sometimes
Rarely
Never

4. Did you feel that you could confide in (talk about important personal things with) your father?

Always
Often
Sometimes
Rarely
Never

5. Was your father available to spend time with you in activities?

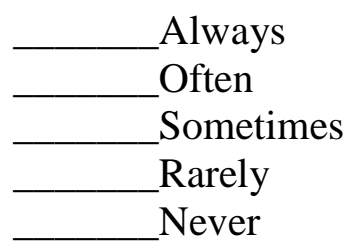

6. How emotionally close were you to your father? 
Extremely close

Very close

Somewhat close

A little close

Not at all close

7. When you were an adolescent (teenager), how well did you get along with your father?

\begin{tabular}{l} 
Very well \\
\hline Well \\
Ok \\
Poorly \\
Very poorly
\end{tabular}

8. Overall, how would you rate your father?

Outstanding

Very good

Good

Fair

Poor

9. As you go through your day, how much of a psychological presence does your father have in your daily thoughts and feelings?

Always there

Often there

Sometimes there

Rarely there

Never there 


\section{Appendix F}

\section{HOW I TYPICALLY ACT TOWARDS MYSELF IN DIFFICULT TIMES}

Please read each statement carefully before answering. To the left of each item, indicate how often you behave in the stated manner, using the following scale:

Almost never

1
2
3
Almost always

4

1. I'm disapproving and judgmental about my own flaws and inadequacies.

2. When I'm feeling down I tend to obsess and fixate on everything that's wrong.

3. When things are going badly for me, I see the difficulties as part of life that everyone goes through.

4. When I think about my inadequacies, it tends to make me feel more separate and cut off from the rest of the world.

5. I try to be loving towards myself when I'm feeling emotional pain.

6. When I fail at something important to me I become consumed by feelings of inadequacy.

7. When I'm down and out, I remind myself that there are lots of other people in the world feeling like I am.

8. When times are really difficult, I tend to be tough on myself.

9. When something upsets me I try to keep my emotions in balance.

10. When I feel inadequate in some way, I try to remind myself that feelings of inadequacy are shared by most people.

11. I'm intolerant and impatient towards those aspects of my personality I don't like. 
12. When I'm going through a very hard time, I give myself the caring and tenderness I need.

13. When I'm feeling down, I tend to feel like most other people are probably happier than I am.

14. When something painful happens I try to take a balanced view of the situation.

15. I try to see my failings as part of the human condition.

16. When I see aspects of myself that I don't like, I get down on myself.

17. When I fail at something important to me I try to keep things in perspective.

18. When I'm really struggling, I tend to feel like other people must be having an easier time of it.

19. I'm kind to myself when I'm experiencing suffering.

20. When something upsets me I get carried away with my feelings.

21. I can be a bit cold-hearted towards myself when I'm experiencing suffering.

22. When I'm feeling down I try to approach my feelings with curiosity and openness.

23. I'm tolerant of my own flaws and inadequacies.

24. When something painful happens I tend to blow the incident out of proportion.

25. When I fail at something that's important to me, I tend to feel alone in my failure.

26. I try to be understanding and patient towards those aspects of my personality I don't like. 


\section{Appendix G}

\section{Scatterplot}

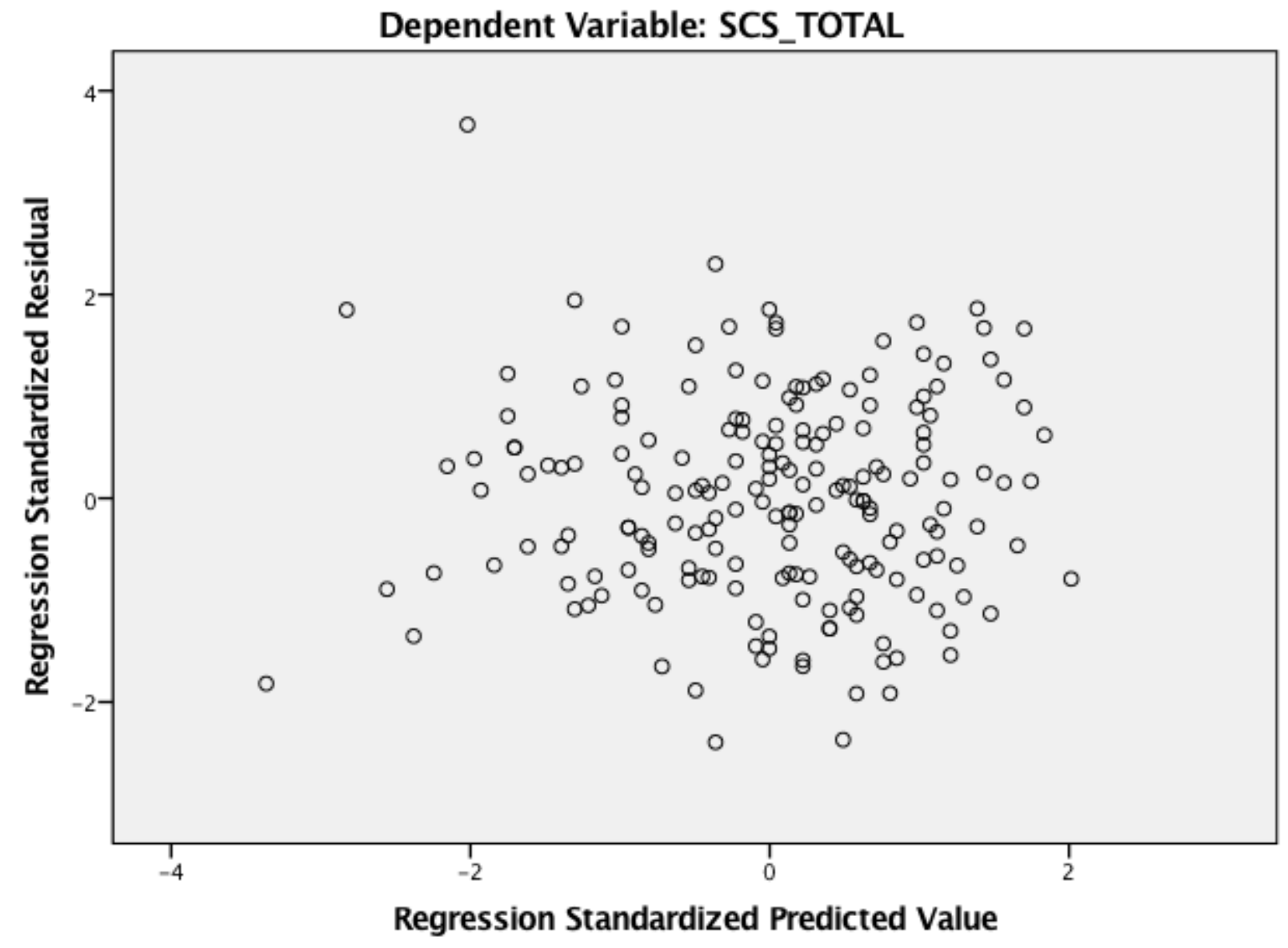




\section{Appendix H}

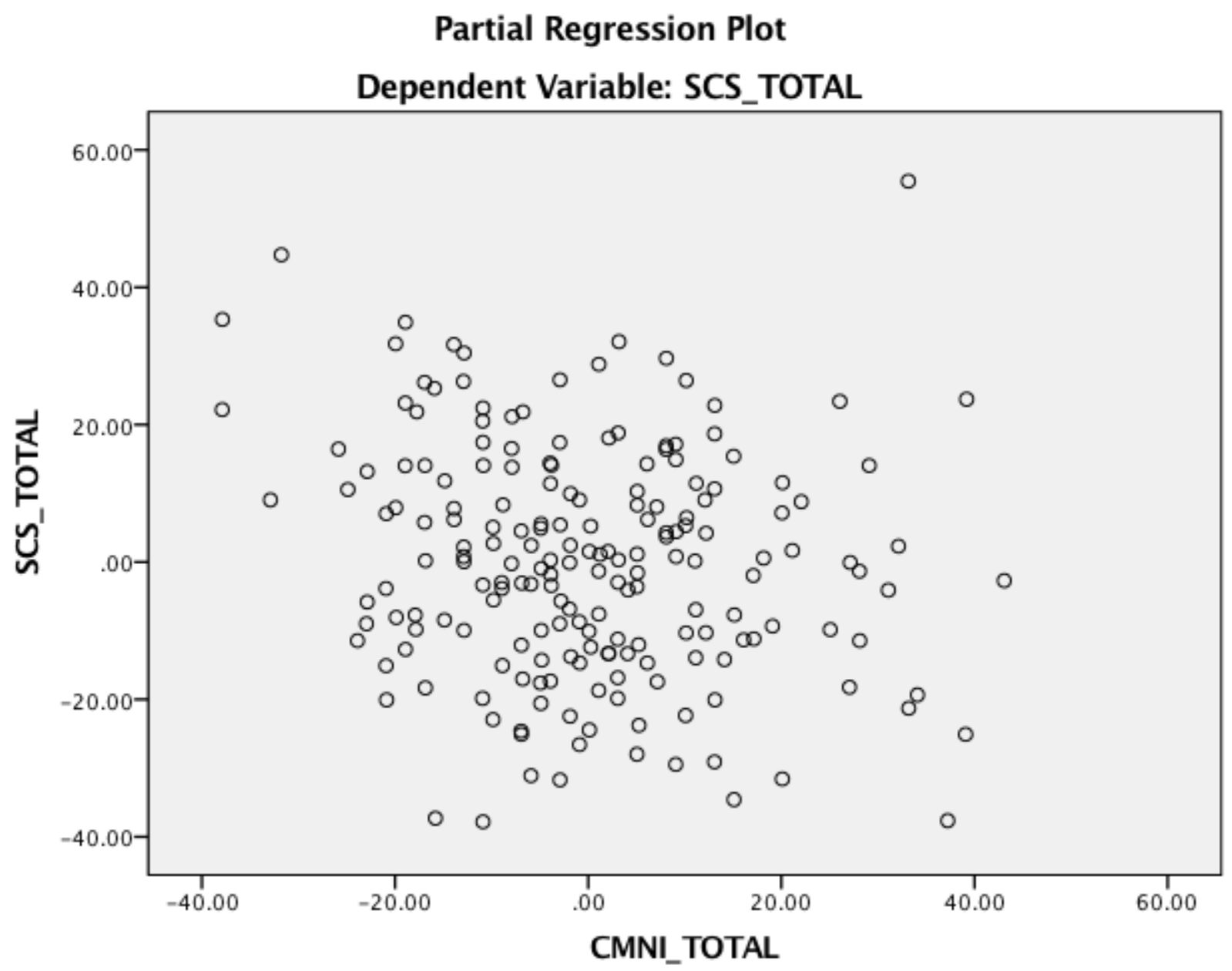




\section{Appendix I}

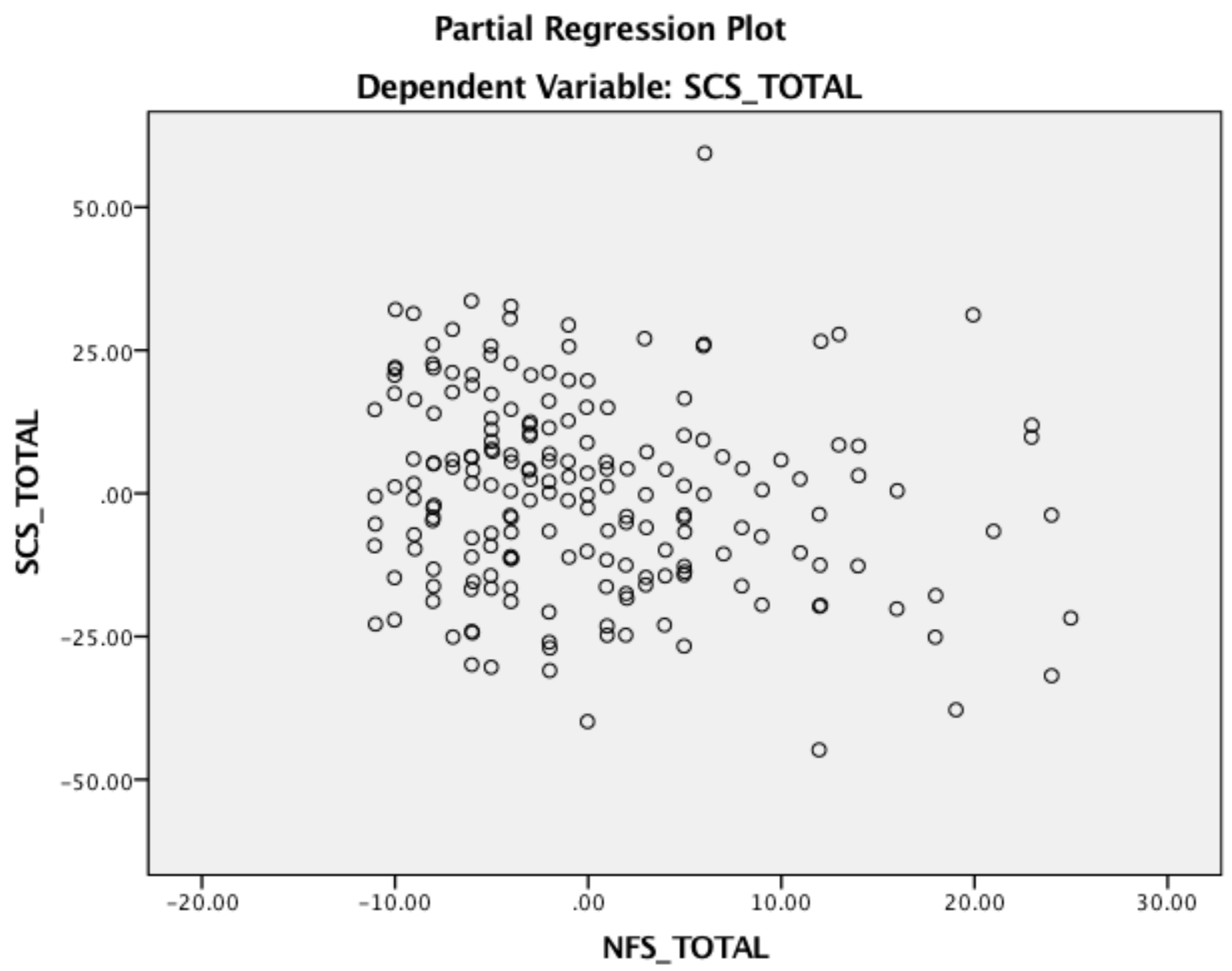

